\title{
Projekt- und Teamarbeit in der digitalisierten Arbeitswelt
}

\section{Susanne Mütze-Niewöhner, Erich Latniak, Thomas Hardwig, Manuel Nicklich, Winfried Hacker, Markus Harlacher, Ulrike Pietrzyk und Simone Kauffeld}

Die Beweggründe, sich aktuell mit Projekt- und Teamarbeit zu befassen, sind vielfältig. Hinsichtlich der Verbreitung von Teams ermittelte die Beschäftigtenbefragung

S. Mütze-Niewöhner $(\bowtie) \cdot$ M. Harlacher

Institut für Arbeitswissenschaft, RWTH Aachen University, Aachen, Deutschland

E-Mail: s.muetze@iaw.rwth-aachen.de

M. Harlacher

E-Mail: m.harlacher@iaw.rwth-aachen.de

E. Latniak

Institut Arbeit und Qualifikation (IAQ), Universität Duisburg-Essen, Duisburg, Deutschland

E-Mail: erich.latniak@uni-due.de

T. Hardwig

Kooperationsstelle Hochschulen und Gewerkschaften, Georg-August-Universität Göttingen,

Göttingen, Deutschland

E-Mail: thardwi@gwdg.de

M. Nicklich

Institut für Soziologie, FAU Erlangen-Nürnberg, Nürnberg, Deutschland

E-Mail: manuel.nicklich@fau.de

W. Hacker · U. Pietrzyk

Technische Universität Dresden, Dresden, Deutschland

E-Mail: winfried.hacker@tu-dresden.de

U. Pietrzyk

E-Mail: ulrike.pietrzyk@tu-dresden.de

S. Kauffeld

Abteilungs für Arbeits-, Organisations- und Sozialpsychologie, Institut für Psychologie,

Technische Universität Braunschweig, Braunschweig, Deutschland

E-Mail: s.kauffeld@tu-braunschschweig.de 
des European Working Conditions Surveys 2015 (Eurofound 2015), dass in Deutschland $53 \%$ der Befragten in einer Form von Teamarbeit tätig sind. In der Repräsentativumfrage zum DGB-Index Gute Arbeit 2016 gaben $33 \%$ der Beschäftigten an, mit verschiedenen Personen über das Internet an gemeinsamen Projekten zu arbeiten (Institut DGB-Index Gute Arbeit 2016). Damit sind Projekt- und Teamarbeit für große Teile der Beschäftigten relevante Organisationsformen von Arbeit und müssen damit - quasi zwangsläufig - Gegenstand arbeitswissenschaftlicher Analyse und Gestaltung sein. Mögliche negative Auswirkungen dieser Arbeitsorganisationsformen auf die Beschäftigten sind zu ermitteln und durch korrigierende Maßnahmen zu vermindern, wenn möglich zu beseitigen oder besser noch durch eine proaktive Arbeitsgestaltung zu vermeiden.

Nun sind teambasierte Formen der Arbeitsorganisation schon seit vielen Jahren Gegenstand der arbeitsbezogenen Forschung und es liegen sowohl Modelle und Konzepte als auch Instrumente und Kriterien vor, die für die Analyse, Bewertung und motivations-, lern- und gesundheitsförderliche Gestaltung herangezogen werden können (z. B. Antoni 2000, 2016; Gerlmaier und Latniak 2007, 2011; Jöns 2008; Hacker 1994; Kauffeld 2001; Kauffeld et al. 2016; Mütze-Niewöhner et al. 2018; Nordhause-Janz und Pekruhl 2000). Allerdings haben sich die Bedingungen, unter denen kooperative Arbeit in Teams stattfindet, in den letzten Jahren durch die fortschreitende Digitalisierung wahrnehmbar verändert und sie verändern sich noch.

Ein starker Beweggrund verbirgt sich also im zweiten Teil des Titels dieses einleitenden Beitrags: Es ist an der Zeit, sowohl die Veränderungen der Arbeitsund Betriebsorganisation als auch die mit der Digitalisierung verbundenen Herausforderungen für die Gestaltung, Organisation, Führung und Regulierung von kooperativer Arbeit in Teams zu erkunden, kritisch zu diskutieren und bestehende Konzepte und Instrumente anzupassen bzw. zu ergänzen. Denn - womit ein dritter Beweggrund benannt ist - Arbeiten in Teams birgt zahlreiche Potenziale, sowohl für die Effektivität und Effizienz als auch für die Qualität von Arbeit und ihrer Bedingungen:

Durch die Zusammenführung von Menschen mit unterschiedlichen Denkweisen, Kompetenzen und Interessen eröffnen sich Chancen, mindestens ebenso vielfältige Ideen zu generieren oder auch - je nach Aufgabenstellung - zu abgestimmten, bedarfsgerechten und in der Folge akzeptierte(re)n Lösungen zu gelangen. ${ }^{1}$ Kommunikation, Interaktion und Zusammenarbeit führen zu variierenden Anforderungen und damit Lern- und Entwicklungsmöglichkeiten. Teamarbeit schafft Optionen, Handlungsspielräume gezielt zu erweitern und das Ausmaß an erlebter Verantwortung und Autonomie zu erhöhen. Eingespielte und gut funktionierende Teams gelten als besonders flexibel und reaktionsschnell, z. B. bei auftretenden Änderungen und extern verursachten Schwankungen, und sie sind (unter Rahmenbedingungen, die entsprechendes Verhalten begünstigen) auch in der Lage,

\footnotetext{
${ }^{1}$ Die Befundlage zur Zusammensetzung von Teams und ihrer Auswirkungen auf die Ergebnisse von Teamarbeit ist differenziert und zum Teil heterogen. Hier ist u. a. auf Joshi und Roh (2009), Ries et al. (2016), Stahl et al. (2010), Stewart (2006) zu verweisen.
} 
teaminterne Schwankungen der Leistungsfähigkeit auszugleichen, indem sich Teammitglieder gegenseitig unterstützen. Stehen adäquate Informations- und Kommunikationstechnologien sowie Kollaborationswerkzeuge zur Verfügung, kann Teamarbeit auch räumliche und zeitliche Barrieren zumindest zeitweise überwinden.

In einer Arbeitswelt, die durch Vielfalt und Varianz, durch Unschärfe und Unsicherheit, durch komplexe Aufgaben- und Problemstellungen, durch eine hohe Veränderungsdynamik und nicht zuletzt durch zunehmende Digitalisierung und Vernetzung geprägt ist, ist gut gestaltete Teamarbeit letztlich unverzichtbar. Insbesondere für die Bewältigung neuartiger oder komplexer Aufgaben- und Problemstellungen können Projekte einen temporären, organisatorischen Rahmen für Teamarbeit geben. Allerdings ist kooperative Arbeit - gerade im Rahmen von (teilweise) virtueller Projektarbeit - mit spezifischen Belastungen verbunden, die bei ungünstigen Rahmenbedingungen oder in Kombination mit anderen Belastungsfaktoren, wie z. B. hohem Zeitdruck, Zielkonflikten oder Kapazitätsengpässen, negative Auswirkungen auf die Leistungsfähigkeit und -bereitschaft der Beteiligten und damit auch auf Qualität und Ergebnisse von Teamarbeit haben können.

Vor diesem Hintergrund geht es aus Sicht der Autor*innen weder darum, Projekt- und Teamarbeit zu verherrlichen, noch sie zu verteufeln: Es gilt vielmehr, einen analytischen Blick auf die betrieblichen Bedingungen, unter denen sie stattfindet, zu werfen, die Herausforderungen zu identifizieren und Empfehlungen für die Gestaltung von Projektoder Teamarbeit abzuleiten. Ziel muss es sein, die organisatorischen, technischen und personalen Bedingungen so zu gestalten, dass Teamarbeit ihre Potenziale - sowohl für Unternehmen als auch die beteiligten Menschen - bestmöglich entfalten kann.

Für die weitere Gestaltung der Team- und Projektarbeit gilt es zu berücksichtigen, dass verschiedene ökonomische, technische und gesellschaftliche Entwicklungen aktuell zusammenlaufen und zu veränderten Bedingungen für die betrieblichen und überbetrieblichen Gestaltungsprozesse führen. Dabei werden bezogen auf die Arbeitsinhalte in diesem Band exemplarisch Teams fokussiert, in denen die Beteiligten vorrangig anspruchsvolle geistige, informatorisch-mentale Arbeit leisten, wie z. B. Wissens- oder Innovationsarbeit, Produkt- oder Softwareentwicklung.

Wir wollen mit diesem Band Beiträge für eine Zwischenbilanz liefern: Wie ist der Stand der Projekt- und Teamarbeit in der digitalisierten Arbeitswelt heute und vor welchen Herausforderungen steht in diesem Zusammenhang die Arbeitsgestaltung?

Für die Beantwortung dieser beiden Fragen gehen wir in vier Schritten vor:

1. Wir blicken zunächst auf die Entwicklung der Projekt- und Teamarbeit zurück, um wesentliche Entwicklungslinien, die für das Verständnis der Gestaltung dieser beiden Arbeitsformen von wesentlicher Bedeutung sind, kurz zu skizzieren.

2. Wir benennen dann auf dieser Grundlage zentrale Herausforderungen, vor denen eine auf die produktive, innovative und nachhaltig menschengerechte Gestaltung der Projekt- und Teamarbeit heute steht.

3. Wir gehen anschließend auf die normativen Grundlagen der Gestaltung solcher Prozesse und Tätigkeiten ein, und skizzieren, in welche Richtung eine Arbeits- und 
Organisationsgestaltung weiterentwickelt werden könnte, um Antworten auf die benannten Herausforderungen zu erarbeiten.

4. Schließlich werden die Beiträge vorgestellt, die sich im Rahmen des Förderschwerpunkts „Arbeit in der digitalisierten Welt" mit diesen Herausforderungen beschäftigt haben und ihre Ergebnisse in diesem Buch präsentieren.

\subsection{Ein Blick zurück und nach vorn...}

Nach einer begrifflichen Klärung wollen wir den Zusammenhang von Projekt- und Teamarbeit und Digitalisierung wenigstens ansatzweise skizzieren. Dabei soll den verfügbaren Definitionen von Team- oder Gruppenarbeit nicht noch eine weitere hinzugefügt, sondern ein Rahmen für die Beiträge dieses Bandes gesetzt werden, der für die unterschiedlichen professionellen und methodischen Zugänge anschlussfähig ist.

Im Folgenden verstehen wir unter Teamarbeit eine Form der Arbeitsorganisation, bei der ein Arbeitsauftrag an eine Gruppe von mindestens drei Arbeitspersonen übertragen, von diesen als gemeinsame Arbeitsaufgabe verstanden, akzeptiert und schließlich kooperativ bewältigt wird (Mütze-Niewöhner et al. 2018). Das Team muss sich darüber verständigen und - je nach Autonomiegrad - selbst oder mit Unterstützung einer Führungskraft steuern und organisieren, wie die gestellten oder partizipativ entwickelten Ziele erreicht werden sollen. Kooperation, Autonomie und Partizipation sind damit zentrale Dimensionen der Auslegung und Gestaltung von Team- bzw. Gruppenarbeit (vgl. Nordhause-Janz und Pekruhl 2000; s. auch Grote 1997; Weber 1999; Wegge 2004; Schattenhofer 2006). Die Begriffe ,Gruppe“ und ,Team‘ werden im Folgenden synonym verwendet, da eine trennscharfe Abgrenzung schwierig erscheint (Antoni 2017; MützeNiewöhner et al. 2018).

Teamarbeit verlangt einen Arbeitsauftrag, der Kooperation erfordert, sowie Ausführungsbedingungen, die die Zusammenarbeit und die hierzu erforderliche Kommunikation (persönlich bzw. digital vermittelt) ermöglichen, bestenfalls fördern, insbesondere durch angemessene Handlungs- und Entscheidungsspielräume, ausreichende zeitliche und materielle Ressourcen sowie geeignete Arbeitsmittel und Unterstützungssysteme (Hacker 1994; Mütze-Niewöhner et al. 2018). Die Teammitglieder können, müssen jedoch nicht zur gleichen Organisation gehören und die Teamzusammensetzung kann im Verlauf der Zusammenarbeit wechseln („Fluidität“) (Wageman et al. 2012; Kauffeld und Schulte 2019).

Projektarbeit wird im Weiteren als eine temporäre Form der Teamarbeit verstanden (Braun und Sydow 2017). Dabei werden Projektteams für die Bearbeitung von befristeten Projektaufträgen zusammengestellt, insbesondere dort wo unterschiedliche Kompetenzen und Expertise von Spezialist*innen zur Lösung eines komplexen, dynamischen und bisher ungelösten (,innovativen“) Problems notwendig sind. Ein Projektteam wird nach Erfüllung seiner Aufgabe aufgelöst oder mit einem neuen Projekt betraut. 
Projektaufgaben sind im Gegensatz zu Routineaufgaben durch Einmaligkeit und Unsicherheit gekennzeichnet. Es ist zunächst unklar, wie die Aufgabe konkret zu erfüllen ist und ob das für den Projekterfolg erforderliche Wissen und die Ressourcen verfügbar gemacht werden können. Diese Klärungen sind wesentlicher Teil der Projektarbeit selbst. Entsprechend lassen sich die Arbeitsleistungen nicht vorab im Detail und in ihrer Reihenfolge definieren und planen, weshalb dem Projektteam eine relativ weitreichende Autonomie bei der Arbeitsplanung und -ausführung eingeräumt werden muss. Aus diesen Merkmalen entstehen nicht nur Unschärfen bei der Kategorisierung der vielfältigen empirischen Erscheinungsformen von Projektarbeit (Kalkowski und Mickler 2009; Mütze-Niewöhner et al. 2018), sondern es gibt vor allem große Differenzen in der konkreten, resultierenden Arbeitssituation der Beteiligten.

Digitalisierung verstehen wir im Anschluss an Hirsch-Kreinsen (2015) als den „Prozess des sozio-ökonomischen Wandels [...], der durch Einführung digitaler Technologien, darauf aufbauender Anwendungssysteme und vor allem ihrer Vernetzung, angestoßen wird“ (Hirsch-Kreinsen 2015, S. 10). Für unseren Zusammenhang ist hier zunächst zu ergänzen, dass sich dies einerseits in und zwischen Betrieben abspielt, andererseits die IT-technische Vernetzung auch eine digital vermittelte Vernetzung von Menschen ermöglicht. Durch die Verfügbarkeit von neuen, digitalen Technologien schafft das Management in den Unternehmen neue, andere Möglichkeiten, Arbeit zu definieren, ausführen zu lassen und zu organisieren. Dabei wird die Arbeit auf drei Ebenen neu geteilt: Verändert wird 1) die Arbeitsteilung zwischen den technischen Systemen, die definierte Funktionen bereitstellen, und den Nutzenden, die wiederum mit veränderten Aufgaben, Anforderungen und Rollen konfrontiert sind. Verändert wird 2) die Arbeitsteilung zwischen unterschiedlichen Beschäftigten/-gruppen, die z. B. in globalen Wertschöpfungs- oder Innovationsprozessen über nationale und organisatorische Grenzen hinweg kooperieren können: Telekommunikationsinfrastrukturen und mobile Endgeräte eröffnen vielfältige Möglichkeiten der Zusammenarbeit über Orts- und Zeitgrenzen hinweg, prototypisch bei der Softwareentwicklung. Verändert wird auf derselben Grundlage schließlich 3) die Aufteilung zwischen produzierenden bzw. dienstleistenden Unternehmen und deren jeweiligen Kunden und Lieferanten (vgl. Gerlmaier und Latniak 2019).

Neu ist aus unserer Sicht an der aktuellen Digitalisierungsphase zudem, dass sich jetzt ein großer Teil der Kommunikation der Beteiligten über digitale Medien vermittelt abspielt, was die unterstützenden Technologien zunehmend in den Fokus rückt (vgl. Pasmore et al. 2018). Dadurch kann die Zusammenarbeit räumlich verteilt in virtuellen Teams (Boos et al. 2017) oder auch in virtuellen Organisationsformen (Warner und Witzel 2004) erfolgen. Bestimmte Tätigkeiten lassen sich, wie das Beispiel Crowdwork illustriert, mit Hilfe von Internetplattformen zunehmend auch ohne Betrieb organisieren (Leimeister et al. 2016).

Diese neuen technologischen Möglichkeiten erzeugen neue Anforderungen und Belastungen, deren Erforschung noch nicht als abgeschlossen gelten kann (MützeNiewöhner und Nitsch 2020). Im Hinblick auf Partizipation und Kooperation eröffnen 
sie für die Beschäftigten ein Potenzial neuer Handlungs- und Entscheidungsspielräume; zum anderen ergeben sich direkte Wirkungen durch die Digitalisierung von Arbeitsmitteln und Arbeitsgegenständen für die Beschäftigten in der Projekt- und Teamarbeit. So verbreiteten sich virtuelle Teams schon seit den 1990er Jahren; sie sind heute allgegenwärtig und prägen zunehmend die Sicht auf Projektarbeit. ${ }^{2}$ Dies illustriert, dass Projekt- und Teamarbeit in vielfältiger Weise und schon seit geraumer Zeit durch Digitalisierungsprozesse in der Arbeitswelt berührt wird.

Vieles, was heute in der digitalen Transformation für die Gestaltung produktiver, innovativer und nachhaltig menschengerechter Arbeit in der Projekt- und Teamarbeit von Bedeutung ist, greift auf Prozesse zurück, die sich seit langem vorbereiten und durchsetzen. Diese Entwicklung skizzieren wir in den drei folgenden Abschnitten, die jeweils verschiedene Entwicklungslinien auf Teams basierender Formen der Arbeitsorganisation beleuchten: die Projektarbeit, die sich zunehmend verbreitet, Gruppenarbeit in der Produktion sowie Teamarbeit in Dienstleistungsbereichen.

\subsubsection{Entwicklung der Projektarbeit}

Als Ursprünge der modernen Projektarbeit und des Projektmanagements werden zumeist das Manhattan-Projekt im 2. Weltkrieg, die Fabrikplanung bei DuPont in den 1950er Jahren (mit der Critical Path Method u. a. zur Kostenkontrolle), die Entwicklung der Polaris-Trägerraketen in den USA seit 1958 (mit der dabei verwendeten ,Program Evaluation and Review Technique، (PERT)) sowie das Apollo-Mondflug-Programm ab 1960 angeführt (vgl. Snyder 1987; Seymour und Hussein 2014). In seinen Anfängen hatte das Projektmanagement eine stark ingenieurwissenschaftliche Ausrichtung mit dem Interesse einer möglichst ,genauen Planung und Steuerung der Arbeitsprozesse“ (Kalkowski 2013, S. 400). Bereits seit den 1960er Jahren findet auch eine Institutionalisierung der Methodenentwicklung und Professionalisierung und damit eine Standardisierung und Normierung des Projektmanagements statt (Muzio et al. 2011). Dies geschah mit dem Ziel, die o. g. grundlegende Unsicherheit und Komplexität der zu bearbeitenden Prozesse und Aufgaben transparent und beherrschbar zu machen. Branchen, die sich auf einmalige Leistungen konzentrieren, bilden dabei oft ,projektbasierte Arbeitsorganisationen“ aus, in denen diese Arbeitsform ganze Unternehmen

\footnotetext{
${ }^{2}$ So stellten Akin und Rumpf (2013) im Rahmen einer nicht repräsentativen Führungskräftebefragung fest, dass $75 \%$ der Unternehmen (Hauptsitz in Deutschland) bzw. $81 \%$ (Hauptsitz im Ausland) virtuelle Teams nutzten; ähnliche Daten bei Manager Monitor (2017). Auf die Erhebung zum DGB-Index 2016 wurde bereits zu Beginn des Beitrags verwiesen. Hier sei ergänzt, dass der Anteil der Beschäftigten, die digital vermittelt in Projekten mit anderen Personen kooperieren (insgesamt $33 \%$ der Befragten), in Abhängigkeit der Branche deutlich variiert (von $11 \%$ im Gastgewerbe bis zu $79 \%$ im Bereich Information und Kommunikation). (Institut DGB-Index Gute Arbeit 2016).
} 
prägt; neben Sondermaschinenbau und Bauwirtschaft sind dies etwa klassische Branchen der Wissensarbeit wie Werbe-, Design- und Eventagenturen, IT-Services oder Beratung.

Als arbeits- und kundenbezogene Gegenbewegung $\mathrm{zu}$ weit verbreiteten bürokratischen Tendenzen im Projektmanagement (Hodgson 2004), hat sich 2001 eine Gruppe von Software-Entwicklern formiert, die sich mit ihrem ,agilen Manifest` von der nunmehr als ,klassisch“ verstandenen Projektarbeit distanzierten und eine stärkere Orientierung an Kunden, teambasierter Selbstorganisation und Arbeitsgegenständen forderten. Aufgegriffen wurde dabei, dass bei Projektarbeit häufig nicht plan- und kalkulierbare Ereignisse eintreten, die zu Abweichungen von der Planung zwingen und teilweise zum Scheitern führen (Heidling 2018). Dies soll durch ein iteratives Vorgehen mit agilen Methoden aufgefangen und produktiv genutzt werden. ${ }^{3}$

Die Gestaltung der Projektarbeit, d. h. der Projektaufgaben, der Verantwortung und Kompetenzen, der Prozesse der Zusammenarbeit, des Methodeneinsatzes und der Beziehungen zu anderen Teilen der Organisation bzw. dem Unternehmensumfeld, ist Gegenstand von Aushandlungsprozessen mit Auftraggeber*innen und Entscheider*innen des Projektes (z. B. Lenkungsausschuss), zwischen Projektleiter*innen und Projektmitarbeiter*innen, zwischen Projektmitarbeiter*innen und ihren Vorgesetzten sowie im Projektteam (vgl. Kötter 2002). Zu einem erheblichen Teil hängt der Erfolg wegen der nicht völlig planbaren Abläufe und Situationen von informellem Arbeitshandeln im Projekt ab (Braun 2018). Von entscheidender Bedeutung für den Projekterfolg wie für die Arbeitsbedingungen ist dabei das Verhältnis der Projekte zur Organisation, in die sie eingebettet sind (Heidling 2018), und der Grad der Entscheidungs- und Handlungsautonomie, der den Projektbeteiligten zugestanden wird.

Die Ansprüche an die Beschäftigten in den Projekten resultieren vor diesem Hintergrund häufig aus internen wie externen „Verständigungsleistungen“ (Kalkowski und Mickler 2015). Dementsprechend ist das Arbeitshandeln in Projekten „sehr viel stärker auf Kooperation und Kommunikation unterschiedlicher Funktionsbereiche und Abteilungen [in Unternehmen, d. V.] ausgerichtet, die in ihren Prozessen traditionell getrennt voneinander agieren..." (Heidling 2018, S. 213). Gerade weil oft bei Projektarbeit in Unternehmen Elemente von bürokratischer, hierarchischer Kontrolle weiterwirken (Hodgson 2004), liegen in dieser Konstellation Quellen der Belastung für Beschäftigte: Unklarheiten in Bezug auf verfügbare Kapazität, Rollen oder Entscheidungs- und Weisungsbefugnisse erzeugen widersprüchliche Anforderungen an Mitarbeiter*innen wie Führungskräfte (Hodgson 2004; Pfeiffer et al. 2014). Dabei

\footnotetext{
${ }^{3}$ Dabei wird von arbeitssoziologischer Seite festgestellt, dass ,,agile Methoden unter bestimmten Voraussetzungen als Schutzraum gegenüber neuen Belastungstypen und freiwilliger Selbstausbeutung wirken können - ein Schutzraum aber, der fragil und ohne interessenpolitisch flankierte Ressourcenkonflikte auf Dauer wohl nicht zu sichern ist." (Pfeiffer et al. 2014, S. 119) Andere kritisieren agile Methoden als Management-Methode ohne nachhaltigen Effekt (Cram und Newell 2016) bzw. eine neue Form von verstärkter Kontrolle der Beschäftigten (Hodgson und Briand 2013; Moore 2018).
} 
können höhere Grade an Handlungsautonomie im Zusammenwirken mit fixen Terminen auch zu Belastungen für die Beschäftigten führen, wie etwa Zeitdruck, Arbeitsunterbrechungen oder ungeplantem Zusatzaufwand, den Klärungsprozesse mit sich bringen (Gerlmaier und Latniak 2013).

Projektarbeit ist zwar nicht technisch bestimmt, doch spielte die Digitalisierung des Projektmanagements auf den damals verfügbaren Großrechnern schon seit der Entwicklung und Nutzung von PERT eine bedeutende Rolle. Bei der Verbreitung und Standardisierung des Projektmanagements wurde die ab Mitte der 1980er Jahre auf PCs einsetzbare Projektmanagementsoftware zu einem wichtigen Verbreitungsfaktor: Sie unterstützt in unterschiedlicher Weise die komplexe Modellierung der Aufgaben- und Ablaufstruktur und die Darstellung der zeitlichen und sozialen Abhängigkeiten. Unterstützung für die Projektarbeit leistet auch die weitere Entwicklung der Informationsund Kommunikationstechnologie: Die Entwicklung des Internets und entsprechender „Groupware“-Anwendungen unterstützen die räumlich verteilte Zusammenarbeit, vergrößern aber auch das Spannungsverhältnis zur klassischen, hierarchischen Organisation (vgl. Lipnack und Stamps 1998).

Insgesamt deutet sich für die Entwicklung der Projektarbeit ein vermittelter Einfluss der Digitalisierung an: Zwar werden einerseits Arbeitsmittel (z. B. ProjektmanagementSoftware, Kommunikationstechnologie) und Arbeitsgegenstände (wie z. B. verteilte CAD-Systeme oder Software-Entwicklungsumgebungen) der Projektarbeit digitalisiert und zudem die Vernetzung der Teams über das Internet vorangetrieben. Andererseits fördern die zunehmende Unsicherheit, Dynamik und Komplexität der wirtschaftlichen Aktivitäten insgesamt den Bedarf an und die Nutzung von effektiverer, digitaler Unterstützung der Projektarbeit.

Mittlerweile gibt es vielfältige Belege dafür, dass sich das Arbeiten in Projekten zunehmend verbreitet (Braun und Sydow 2017). Rump et al. (2010) kamen zu der Einschätzung, dass $37 \%$ aller Arbeitsabläufe projektwirtschaftlich organisiert seien. Sie beobachteten, dass in etwa $30 \%$ der Unternehmen die Mehrzahl der Beschäftigten in Projekten eingesetzt würde. Aktuellere Befunde ergeben, dass - mit großen Branchenunterschieden - 34,7\% der Gesamtarbeitszeit in Deutschland als Projekttätigkeit geleistet wird und sich gegenüber 2009 eine Steigerung (29,3\%) ergeben hat (GPM 2015).

Insbesondere durch zunehmende Tendenzen von Dezentralisierung, Translokalisierung und Internationalisierung hat sich die räumlich verteilte Zusammenarbeit in Projekten etabliert: So arbeiten Projektbeteiligte nur noch zu $20 \%$ im selben Raum, die überwiegende Mehrheit damit also räumlich verteilt, und zwar zu $43 \%$ in unterschiedlichen Bereichen am selben Standort, zu $28 \%$ an verteilten Standorten (Rump et al. 2010).

Drei aktuelle Entwicklungen, die mit Digitalisierungsaspekten in Verbindung stehen, kennzeichnen Projektarbeit derzeit: 1) das Herauslösen von Projekten aus betrieblichen Strukturen und die plattformvermittelte Kooperation, etwa im Zusammenhang mit Crowdwork, 2) das ,Öffnen“ nach außen und der Einbezug von Nutzenden bzw. 
Kund*innen, z. B. bei Open Innovation-Prozessen, und 3) die Nutzung agiler Methoden im Projektmanagement (Pfeiffer et al. 2014). Diese Trends, so sie sich durchsetzen, stellen die Gestaltung von Projektarbeit vor verschärfte und neue Herausforderungen, auf die geeignete Antworten gefunden werden müssen.

\subsubsection{Team- und Gruppenarbeit in der Produktion}

Teams und Gruppen in produzierenden Unternehmen werden in unterschiedlichen Bereichen genutzt: Während in IT- oder Entwicklungsbereichen und im Vertrieb häufig in Projekten gearbeitet wird (vgl. Rump et al. 2010), sind in der Produktion auf Dauer angelegte Teams weit verbreitet, die z. T. durch temporäre Teamstrukturen ergänzt werden (z. B. Qualitätszirkel oder KVP-Gruppen).

Für die Gestaltung von Team- bzw. Gruppenarbeit in der Produktion kann auf eine lange Tradition zurückgeblickt werden, die sich von den ersten Untersuchungen zu soziotechnischen Systemen in den 1950er Jahren und den Arbeiten von Kurt Lewin über Gruppen, über Versuche im Rahmen des ,Industrial Democracy'-Programms in Norwegen zurückverfolgen lässt (dazu u. a. Trist und Bamforth 1951; Cherns 1976, 1987; Sydow 1985). Diese Entwicklungen waren über viele Jahre von einer stark antitayloristischen Ausrichtung geprägt (Pruijt 2003). Das tayloristische Konzept der Rationalisierung der Produktion hatte sich im 20. Jahrhundert in der industriellen Produktion nach und nach durchgesetzt. Die Nebenfolgen dieses Rationalisierungskonzepts bestanden zum einen in extrem bürokratischen inflexiblen Produktionsstrukturen, die nur verzögert auf veränderte Wettbewerbsbedingungen reagieren konnten; zum anderen in monotonen Arbeitsbedingungen, die bei den Beschäftigten zu Entfremdungserscheinungen, Motivationsproblemen, erhöhten Krankheitsquoten und Fluktuation führten. In Schweden veranlassten etwa die hohen Fluktuationsquoten im Zusammenspiel mit arbeitsmarktbedingten Engpässen die Entwicklung von weniger monotonen Gruppenarbeitskonzepten (Berggren 1991).

In Deutschland war die Entwicklung durch staatlich geförderte Experimente im Rahmen des Bundesprogramms ,Humanisierung des Arbeitslebens' geprägt, die aber zunächst weder bei den Gewerkschaften noch beim Management auf breite Zustimmung stießen, noch in vielen Unternehmen übernommen wurden. Dennoch konstatierten Kern und Schumann (1986) in den Industrieunternehmen einen sich abzeichnenden Umbruch hin zu ,neuen Produktionskonzepten“. Damit beschrieben sie Arbeitseinsatzkonzepte, mit denen die starren Formen der Massenproduktion durch eine Nutzung von ,Produktionsintelligenz' flexibilisiert werden sollten. Dies sei nötig, weil die zunehmende Automatisierung und die einsetzende Digitalisierung in hochtechnisierten Bereichen (u. a. durch Nutzung von Produktionsplanungs- und Steuerungssystemen, Betriebsdatenerfassung, Computer Aided Design usw.) überwiegend qualifizierte Gewährleistungs-, Überwachungs- und Steuerungsaufgaben übrigblieben, für die nun eine adäquate Arbeitsorganisation zu gestalten wäre (vgl. Kern und Schumann 1986). 
Während Automatisierung und Digitalisierung also in Hochtechnologiebereichen in dieser Zeit tendenziell Raum für Gruppenarbeit schuf (Hirsch-Kreinsen und Ramge 1994), verblieben viele arbeitsorganisatorische Lösungen in Produktions- und Montagebereichen weitgehend im tayloristischen Rahmen, teilweise allerdings für die jeweiligen Teams um dispositive Aufgaben mit begrenzter Autonomie und Selbstorganisation (,Teilautonomie‘), Gruppengespräche und Kommunikation zur betrieblichen Steuerung und Kontrolle der Abläufe angereichert. An Stelle einer zentralen Vorgabe aller Abläufe sollte nun die Feinsteuerung dezentral an sich selbst steuernde Einheiten, eben: teilautonome Produktionsteams, übertragen werden, um so flexibler auf Abweichungen und ungeplante Zustände reagieren zu können (vgl. u. a. Latniak 2013).

Hinzu kamen aus einer ganz anderen Tradition in den 1990er Jahren Impulse zur Veränderung der Arbeit von Teams in der Produktion: Die 1990 veröffentlichte Studie von Womack et al. (1992) verschaffte dem Konzept ,Lean Production“ eine enorme Popularität, das auf die konsequente Vermeidung jeglicher Art von „Verschwendung“ und die ständige Optimierung der Wertschöpfungsprozesse zielte. Insbesondere für den dafür zentralen kontinuierlichen Verbesserungsprozess (Imai 1993) und für das Qualitätsmanagement (z. B. Total Quality Management, vgl. Malorny 2014), das von den Endherstellern ausgehend über die Wertschöpfungsketten (z. B. im AutomotiveBereich) ausgerollt wurde, spielten sog. Lean-Teams eine strategisch wichtige Rolle. Zum einen bekamen sie die Aufgabe übertragen, auftretende Fehler und Störungen zeitnah zu beseitigen, um die Qualitätsziele zu erreichen. Zum anderen wurden von ihnen dabei Verbesserungen der Arbeitsstandards entwickelt, die dann im Betrieb insgesamt umgesetzt wurden, um so Redundanzen und Ressourcen zu reduzieren und zur , schlanken Produktion` beizutragen.

Letztlich entstanden in der Praxis der produzierenden Betriebe vielfältige Formen von Team- und Gruppenarbeit, die sich häufig keinem der angesprochenen Konzepte mehr eindeutig zuordnen lassen, nicht zuletzt weil einerseits bestehende technische wie organisatorische Strukturen in den Unternehmen erst Schritt für Schritt angepasst wurden, zum anderen weil Veränderungen in den Unternehmen nur teilweise oder nicht in allen Bereichen wie vorgesehen umgesetzt werden konnten (s. auch Antoni 2000; Mütze-Niewöhner et al. 2018).

Die Phase des Einführungsbooms von Gruppenarbeit in den 1990er Jahren, die von einer Vielzahl von Veröffentlichungen und arbeitspolitischen Debatten begleitet wurde, endete etwa Mitte der 2000er Jahre (Kirchner und Oppen 2007), gleichwohl in vielen Unternehmen Teams zu einem festen Bestandteil der Organisation geworden sind, ohne dass dies von der Forschung besonders vermerkt wurde. Hinsichtlich der Verbreitung ist davon auszugehen, dass bis 2012 in $49 \%$ der Industriebetriebe in Deutschland eine Form von Gruppenarbeit in der Produktion eingeführt wurde (Som und Jäger 2012).

Durch die aktuelle Phase der Digitalisierung könnte neue Bewegung in die Debatte um Teamarbeit kommen, wie die Diskussion um Industrie 4.0 offenlegt. Der steigende Anteil kundenspezifischer Produkte in kleinen Losgrößen und der intendierte Aufbau von Cyber-physischen Systemen erhöhen die Komplexität der Produktionssysteme, was 
die Frage aufwirft, wie wichtig hierfür ,reaktionsschnelle und mit hohen Autonomiegraden ausgestattete Teams“ (Mütze-Niewöhner et al. 2018, S. 685) sein werden. Aus arbeitsorganisatorischer Sicht bieten die genannten Entwicklungen durchaus Chancen für die Verbreitung von (teil-)autonomen Arbeitsgruppen. Die Einschätzungen, wie weit dies realisiert werden könnte, gehen derzeit allerdings noch weit auseinander. Breiter Konsens besteht hingegen in der Literatur, dass nicht die technologischen Potenziale die weitere Entwicklung der Produktionsarbeit bestimmen, sondern arbeitspolitische Entscheidungen der betrieblichen Akteure und die konkrete Arbeitsgestaltung (MützeNiewöhner und Nitsch 2020; Hirsch-Kreinsen 2015; Boes et al. 2015; Kuhlmann und Schumann 2015; Windelband 2014).

\subsubsection{Teams in der Dienstleistungsarbeit}

Dienstleistungsarbeit und -tätigkeiten sind vielfältig (Minssen 2019) und ,entsprechend vielfältig ist auch die Forschungslage zu Dienstleistungsarbeit“" (Oberbeck 2013, S. 104). Diese Heterogenität sowie das damit zusammenhängende Fehlen eines etablierten (also von der multidisziplinären Dienstleistungsforschung auch angewandten) Klassifizierungsansatzes für Dienstleistungen lassen es derzeit nicht $\mathrm{zu}$, allgemeine Aussagen zu den Entwicklungslinien von Teamarbeit im Dienstleistungsbereich zu treffen. Erschwerend kommt hinzu, dass sich die teambezogene Arbeitsforschung in der Vergangenheit - zumindest in Deutschland - sehr viel intensiver mit der Gruppenarbeit in der Produktion befasst hat. Dies mag zum einen daran liegen, dass Teamarbeit in Dienstleistungsbereichen weniger verbreitet ist als in der Produktion (s. u.), zum anderen aber auch daran, dass in den unterschiedlichen Dienstleistungsbranchen und Tätigkeitsfeldern andere Herausforderungen der Arbeitsgestaltung dringender waren (wie z. B. die Arbeitsbedingungen in Call Centern, in der Logistik oder in der Pflege). Im Weiteren werden wir deshalb eher eklektisch einzelne Aspekte und Gestaltungsbeispiele hervorheben, die für den Betrachtungsgegenstand dieses Bandes relevant sind.

Betrachtet man zunächst die Digitalisierung und Technisierung in der Dienstleistungsarbeit, so boten sich früher vor allem die Büro- und Verwaltungsarbeiten für eine Technisierung an, die als ,Backoffice‘-Tätigkeiten bezeichnet wurden: Dies waren standardisierbare Verwaltungs- und Datenverarbeitungsaufgaben, die heute größtenteils durch Programme erledigt werden. Breit durchgesetzt hatte sich diese elektronische Datenverarbeitung (EDV) seit den 1970er Jahren (vgl. z. B. zum Bankensektor Harmsen et al. 1991).

Digitale Technologien wurden seit den 1980er Jahren zunehmend in vier unterschiedlichen Formen genutzt: zur systematischen Automatisierung der Bearbeitung, in computerunterstützter sowie computergesteuerter Sachbearbeitung sowie in der Bereitstellung von Datenbanken als Management-Informationssystem. Keine dieser Nutzungsformen der Technologie erweitert in relevanter Weise die Kooperationsmöglichkeiten der 
Angestellten. Daher wurde Teamarbeit im Büro in dieser empirischen Studie über verschiedene Branchen auch nicht gefunden (Baethge und Oberbeck 1986).

Als sehr begrenzt rationalisierbar und algorithmierbar gelten (noch) solche dienstleistenden Tätigkeiten, die mit Ermessensspielräumen bei Entscheidungen verbunden sind, wie z. B. die qualifizierte Sachbearbeitung, ebenso wie Aufgaben mit großen Kreativanteilen, z. B. in Entwicklungs- oder Innovationsbereichen. Damit verbunden sind ein größerer Autonomiespielraum für die Ausführung der Tätigkeit, andere Kontrollformen des Managements (,verantwortliche Autonomie“) sowie stärker vertrauensbasierte Sozialbeziehungen (Heisig et al. 1992). Wie dargestellt spielt die Projektarbeit insbesondere in der Entwicklungstätigkeit, im Vertrieb und in weiteren Bereichen der qualifizierten Wissensarbeit eine wichtige Rolle. Mit dem Zuwachs an wissensintensiven Dienstleistungen ist eine weitere Zunahme der Verbreitung von Projektarbeit zu erwarten; greifen die Bemühungen, den Export von wissensintensiven Dienstleistungen anzukurbeln, wird sich gleichzeitig auch der Anteil an verteilter, virtueller Zusammenarbeit erhöhen.

Ein Beispiel aus dem Bereich personenbezogener Dienstleistungsarbeit im Gesundheitswesen illustriert eine Form von Teamarbeit, die als ,interprofessionelle Teamarbeit“ bezeichnet werden kann (Antoni 2010): Ein niederländischer Pflegedienstleister konnte auf Basis von selbstgesteuerten Teams eine marktdominierende Stellung entwickeln. Sowohl günstige Leistungs- als auch Kostengesichtspunkte sowie eine hohe Arbeitszufriedenheit seiner Mitarbeiterinnen und Mitarbeiter sind kennzeichnend für dieses Unternehmen (Nandram und Koster 2014). Technische Voraussetzung für die vollintegrierte Pflege durch selbstgesteuerte lokale Teams ist eine Kollaborationsplattform, über die die administrativen Tätigkeiten und der wechselseitige Austausch und die Unterstützung der Teams organisiert wird.

Aktuelle Überblicksdaten zur generellen Nutzung von (dauerhaften) Teams im Dienstleistungsbereich sind uns nicht bekannt. Lediglich die ältere Beschäftigtenbefragung von Born (2000, auf Basis von 1626 Befragten) liefert für die Bundesrepublik übergreifende Daten für die Verbreitung: Er ermittelte, dass im Dienstleistungssektor $11,5 \%$ der befragten Beschäftigten in Teams arbeiten („Gruppenarbeit insgesamt“), mit höheren Anteilen im Bereich sozialer Dienstleistungen $(15,7 \%)$ und im staatlichen Bereich (13,1\%), gefolgt von den konsumbezogenen (12\%) und produktionsnahen $(11,5 \%)$ Dienstleistungen. Der distributive Bereich (Handel und Logistik) fällt dagegen mit 8,9\% der Befragten in Teams etwas ab.

Offen ist, ob es mit der aktuellen Phase der Digitalisierung insgesamt zu einer „Transformation tertiärer Arbeit durch Technologie“ kommen wird (Staab und Prediger 2019, S. 123). Es ist eine offene Frage, inwieweit es dabei in konkreten Gestaltungsprozessen gelingt, eine Unterstützung der Tätigkeiten durch Technologien zu erreichen, oder ob es zu einer Substitution von menschlicher Arbeit durch IT-Prozesse auch bei dienstleistenden Tätigkeiten kommen wird, die bislang als eingeschränkt algorithmierbar galten. Inwieweit hier durch die Entstehung einer Plattformökonomie bisherige langfristige Entwicklungslinien aufgebrochen werden und völlig neue Bedingungen ent- 
stehen, bleibt zu beobachten. Wie bei der Digitalisierung der Produktionsarbeit wird aber auch hier erkennbar, dass die technologische Entwicklung die praktische Frage nach einer angemessenen Lösung für die Arbeitsorganisation und die Nutzung von Teams aufwirft, ohne die Antworten vorzugeben.

\subsection{Zentrale Herausforderungen für die Gestaltung der Team- und Projektarbeit in der digitalisierten Arbeitswelt}

Der Rückblick hat gezeigt, dass die zunehmende Verbreitung und Nutzung digitaler Technologien keinen unmittelbaren Einfluss auf die Wahl der Arbeitsorganisation gehabt hat und damit weder die Einführung von Teamarbeit noch die Nutzung von Projektarbeit bestimmt hätte. Allerdings haben sich immer wieder Phasen ergeben, in denen die technologische Entwicklung Spielräume für kooperative Arbeit in Teams eröffnet und unterstützt hat. Nicht zuletzt hat die Digitalisierung auch neue Möglichkeiten für räumlich verteilte kooperative Arbeitsformen geschaffen.

Eine weitere Erkenntnis ist: Es gibt keine Sachzwänge, wie die jeweils verfügbare Technologie eingesetzt wird. Es ist vielmehr notwendig, arbeitspolitische Entscheidungen über den Stellenwert zu treffen, den der Mensch bei der Nutzung der Technologie in Zukunft bekommen soll: Soll die Digitalisierung dazu dienen, als Werkzeug und Assistenzsystem für selbstgesteuerte Teams und Projekte zu fungieren, oder soll sie eingesetzt werden, um auf Basis automatischer Entscheidungsprozeduren und technisch festgelegter Prozesse menschliche Arbeit zu kontrollieren und zu bestimmen? Dies kann nicht abstrakt entschieden werden, sondern klärt sich insbesondere in den alltäglichen Arbeits- und Organisationsgestaltungsprozessen. „Entscheidend wird sein, ob es gelingt, die [...] bestehenden Gestaltungsspielräume zu nutzen, um die vielfältigen Potenziale der digitalen Transformation für eine innovative und soziale, sowohl ökonomische als auch menschzentrierte Gestaltung unserer Arbeitswelt [...] auszuschöpfen.“ (Mütze-Niewöhner und Nitsch 2020, S. 1209) Damit gewinnt die betriebliche Arbeitsgestaltung auch für die Zukunft der Arbeit in Teams und Projekten eine zentrale Rolle.

Über diese generelle Erkenntnis hinaus lassen sich heute zunächst vier Herausforderungen für die Arbeitsgestaltung der Projekt- und Teamarbeit in der digitalisierten Arbeitswelt identifizieren, die in den weiteren Beiträgen zu diesem Sammelband detaillierter aufgeschlüsselt und behandelt werden:

1. die Anpassung der Arbeitsorganisation an die Anforderungen einer zunehmend komplexen und dynamischen Umwelt, die sich in veränderten Anforderungen an die Beschäftigten niederschlägt,

2. die Organisation, Führung und Unterstützung von hybriden oder vollständig virtuell kooperierenden Teams, 
3. die Nutzung neuer, digitaler Werkzeuge für eine Verbesserung der Kommunikation und Kooperation sowie der Arbeitsbedingungen in den Teams und darüber hinaus und

4. die Verhandlung von Interessen und die Regelung der Bedingungen digitaler Arbeit in den unterschiedlichen organisatorischen Kontexten.

Zunächst gilt es, sich der normativen Grundlagen und des arbeitswissenschaftlichen Stands der Arbeitsgestaltung zu versichern, um dann vor dem Hintergrund der eben genannten Herausforderungen Entwicklungsperspektiven für die Gestaltung zu entwickeln. Die Arbeit der verschiedenen Verbundprojekte, die in der Schwerpunktgruppe Projekt- und Teamarbeit zusammengefasst sind, hat für die Diskussion um die Zukunft dieser Arbeits- und Organisationsgestaltung einige Aspekte anzubieten, die im Anschluss daran im Überblick vorgestellt werden.

\subsection{Grundlagen der Arbeitsgestaltung in der digitalisierten Arbeitswelt}

\subsubsection{Gestaltungsgegenstände, Ziele und Maßstäbe}

Das Gestalten von Erwerbsarbeitsprozessen bezeichnet einen komplexen, idealerweise präventiven Vorgang, der marktabhängige wirtschaftliche, technische, organisatorische und menschenbezogene Erfordernisse berücksichtigen muss. Dieser Vorgang sollte partizipativ, d. h. mit Betroffenenbeteiligung oder durch die Betroffenen selbst, sowie kontinuierlich erfolgen (Rothe et al. 2019; Sträter 2019); eine Beteiligung an Arbeitsgestaltungsprozessen setzt eine arbeitswissenschaftliche Mindestqualifikation voraus.

Der Begriff Arbeitsgestaltung kann unterschiedlich verstanden werden. „Arbeitsgestaltung bezeichnet im engeren Sinne das Auslegen der Arbeitsaufträge und ihrer Ausführungsbedingungen.“ (Hacker 2017, S. 247) Zu den Gestaltungsgegenständen zählen u. a. der Arbeitsauftrag mit seinen inhaltlichen und zeitlichen Anforderungen, die (gestaltbaren) Leistungsvoraussetzungen der Arbeitspersonen, die Arbeitsgegenstände, die technischen und sozialen Arbeits- und Hilfsmittel, die (gestaltbaren) wirtschaftlichen und organisatorischen Bedingungen sowie die Beschäftigungsverhältnisse. Da die zu bearbeitenden Arbeitsgegenstände in vielen Bereichen zunehmend Informationen und Daten sind, schließt die Arbeitsgestaltung auch die Informationsflussgestaltung (wer benötigt, welche Information wozu, von wem, wann, in welcher Darstellung) mit ihren kognitionswissenschaftlichen Grundlagen ein (Hacker 2018).

Bei innerbetrieblicher Kooperation ist zusätzlich auch die Art dieser Kooperationen (z. B. face-to-face oder IT-vermittelt) für alle Beteiligten zu gestalten. Bei betriebs- bzw. organisationsübergreifender Kooperation ist ein weiterer Gestaltungsgegenstand die Art des digitalen Informationsaustauschs der weltweit am Prozess Beteiligten (Sträter 2019).

„Die Arbeitsgestaltung verfolgt idealerweise mehrere Ziele gleichzeitig: Sie soll bestmögliche Leistungen nach Qualität und Menge sichern, also leistungsförderlich 
sein, sowie zu diesem Zweck auch lernförderlich und mindestens gesundheitserhaltend, besser noch gesundheitsförderlich wirken.“ (Hacker 2017, S. 247) Arbeitswissenschaftlich fundierte Arbeitsgestaltung erfolgt zielgerichtet und bewusst, und grenzt sich damit grundsätzlich ab vom (ggf. unbeabsichtigten) Verändern, Beseitigen oder Erzeugen von Arbeitsprozessen durch technische oder informationstechnische Maßnahmen zur Automatisierung oder Digitalisierung sowie von Managementmaßnahmen (z. B. Einlasten von Projekten mit spezifischen Kooperationsanforderungen) - jeweils ohne das Ziel ihrer menschengerechten Auslegung.

Die in internationalen und nationalen Standards formulierten Ziele der Arbeitsgestaltung sind in zahlreichen gesicherten Befunden verankerte Erfordernisse. Als Mindest- oder Basisforderung verpflichtet das Deutsche Arbeitsschutzgesetz (ArbSchG) zur Gefährdungsermittlung und -beseitigung einschließlich psychischer Gefährdungen (vgl. auch Gemeinsame Deutsche Arbeitsschutzstrategie, BMAS, BDA \& DGB 2013).

Die in internationalen Normen verankerten Forderungen an die Arbeitsgestaltung gehen in ihren Zielen über den Schutz vor Gefährdung noch hinaus: Der Standard DIN EN ISO 6385 (2016) stellt Forderungen zur „Optimierung der Arbeitsanforderungen und das Herbeiführen fördernder Arbeitsauswirkungen“ (S. 10) und formuliert dazu Kernmerkmale leistungs-, lern- und gesundheitsfördernder Arbeitsgestaltung. Er wird vertieft durch DIN EN ISO 10075/1-3 (2018), worin langfristig „förderliche Auswirkungen“ von Arbeit, nämlich Lernprozesse zur Ausdifferenzierung vorhandener Leistungsvoraussetzungen und zu ihrem Neuerwerb neben dem Vermeiden beeinträchtigender Wirkungen benannt sind. Flankierend fordert DIN EN ISO 9241-210 (2019) für das Gestalten moderner Arbeitsmittel und interaktiver Systeme deren menschzentrierte und gebrauchstaugliche Gestaltung von den Systementwicklern unter Beteiligung der späteren Nutzer*innen.

Die Gestaltungsforderungen bilden ein hierarchisches System von Bewertungsebenen der Gestaltungsgüte von Erwerbsarbeitsprozessen. Dieses umfasst Ausführbarkeit, Schädigungslosigkeit (z. B. Vermeiden von Arbeitsunfällen, arbeitsbedingten Erkrankungen oder von mentalem Abbau), Beeinträchtigungslosigkeit (z. B. keine unzumutbare Ermüdung) sowie Lern- und Gesundheitsförderlichkeit (ausführlicher Hacker 1995, Abschn. 9-15; Hacker 2009, Abschn. 12.1).

Diese Forderungen müssen zum Gestalten von Arbeitsprozessen mit Hilfe von Einzelmerkmalen konkretisiert werden. Zentrale Einzelmerkmale für Arbeitsaufträge mit fördernden Auswirkungen sind die Vollständigkeit oder Ganzheitlichkeit (die sich insbesondere aus der Arbeitsteilung ergibt), die Anforderungsvielfalt, inhaltlicher und zeitlicher Spielraum für eigenes Entscheiden, differenzierte Rückmeldungen sowie Kommunikations- und Kooperationsmöglichkeiten. Das Verwirklichen dieser schützenden und fördernden Gestaltungsziele erfordert, bislang ungenügend bewältigte Schwierigkeiten zu überwinden: Die Voraussetzungen schützender und fördernder Arbeitsgestaltung werden beim Entwickeln von Automatisierungs- sowie ManagementLösungen geschaffen oder verfehlt. Das verlangt, dabei mögliche Konsequenzen für die Arbeitsgestaltung zu bedenken, die u. U. noch kaum absehbar sind. 
Des Weiteren sind mögliche dialektische (,janusköpfige“; Höge 2019) Folgen von Gestaltungsentscheidungen $\mathrm{zu}$ berücksichtigen. Beispielsweise gehört $\mathrm{zu}$ fördernder Arbeit auch Autonomie der Arbeitenden, um verantwortliches Handeln und intrinsische Motivation anzuregen. Gleichzeitig ermöglicht Autonomie jedoch Selbstgefährdung (Bredehöft et al. 2015). Arbeitsgestaltung sollte daher die Anregung zum verantwortlichen Umgang mit diesen Freiheiten einschließen.

Projekt- und Teamarbeit bietet in diesem Kontext durchaus das Potenzial, zur Erfüllung der Anforderungen menschengerechter Arbeit beizutragen. Ihre Ausgestaltungsformen müssen sich aber letztlich immer an der resultierenden Qualität der Arbeitssituation der einzelnen Arbeitspersonen - sowohl der Teammitglieder als auch der Führungspersonen - messen lassen.

Eine Ursache der verbesserungswürdigen Qualität von Arbeitsprozessen dürfte sein, dass Arbeitsprozesse im Zuge von Digitalisierungsvorhaben häufig weitgehend unbeabsichtigt und ohne Bezug auf die Merkmale gut gestalteter Arbeit festgelegt werden. Folgenreiche, aber zumeist unbeabsichtigte Wirkungen auf Arbeitsprozesse entstehen beim Entwickeln technischer bzw. informationstechnischer Lösungen, die die Funktionsverteilung zwischen Mensch und Technik festlegen, oder beim Gestalten der inner- und überbetrieblichen Arbeitsorganisation. Impulse zum Überwinden des immer wieder beobachtbaren Technikdeterminismus - wie anthropozentrische Produktionskonzepte (Brödner 1985; Lutz 1987) oder beschäftigtenorientierte Technik- und Organisationsgestaltung als Arbeitshumanisierung (GfA 1999) - hatten bislang wenig Erfolg (Raehlmann 2017).

Die Merkmale und Grundlagen präventiver menschengerechter, d. h. schädigungsund beeinträchtigungsloser sowie leistungs-, lern- und gesundheitsfördernder Arbeitsgestaltung sind weitgehend bekannt. Die praktische Realisierung ist ständig neu erforderlich. Dabei sind in den konkreten Gestaltungsprozessen auch die Möglichkeiten der betrieblichen Mitbestimmung und Mitwirkung durch Betriebs- bzw. Personalräte zu berücksichtigen und zu nutzen, insofern sie für direkte Beteiligung an Gestaltungsprozessen Rahmen und Voraussetzungen bilden und für die Berücksichtigung der arbeitswissenschaftlichen Normen ebenfalls Sorge tragen können.

\subsubsection{Herausforderungen der Projekt- und Teamarbeit für die Arbeits- und Organisationsgestaltung}

Technische und organisatorische Herausforderungen (wie die in 1.2 genannten) tragen dazu bei, dass eine an den in 1.3.1 skizzierten arbeitswissenschaftlichen Kriterien ausgerichtete Gestaltung der Projekt- und Teamarbeit immer weniger von standardisierten und einheitlich regelbaren Tätigkeiten und Arbeitszuschnitten an den unterschiedlichen individuellen Arbeitsplätzen ausgehen kann. Die an den unterschiedlichen Arbeitsplätzen verfügbare Funktionsvielfalt trägt dazu bei, dass Arbeitstätigkeiten nach individualisierten Vorgaben, Präferenzen und Bedarfen erledigt werden. Eine Standardisierung von Arbeit, Tätigkeiten und Abläufen, die für alle Teammitglieder 
gleichermaßen greift und damit gleichzeitig quasi einheitlich für gute Arbeitsbedingungen sorgen könnte, wird zunehmend schwierig. Dennoch wird versucht, die Abläufe in den Teams - quasi auf einer Meta-Ebene - über Standardisierungen z. B. im Rahmen von Scrum-Ansätzen oder über eine indirekte Rahmensteuerung $\mathrm{zu}$ kontrollieren. Für unseren Kontext der Arbeitsgestaltung erscheint es deshalb zweckmäßig, an Konzepte zur differenziellen Arbeitsgestaltung anzuknüpfen (vgl. Ulich 2011) und diese den veränderten Bedingungen anzupassen.

Für die Mehrzahl der in Projektteams Beschäftigten ist etwa davon auszugehen, dass für sie einerseits technisch häufig keine festen Arbeitsabläufe mehr vorgegeben sind, damit also, abhängig von der jeweiligen Planung und Terminsetzung, grundsätzlich Handlungs- und Entscheidungsspielraum in der Ausführung besteht. Andererseits müssen von ihnen in vielen Fällen mehrere Aufgaben nebeneinander oder überlappend bearbeitet werden, deren Reihenfolge sie in den angedeuteten Planungsgrenzen selbst bestimmen können (Mehrstellenarbeit). Zudem sind durch die verwendeten Software-Tools oft äquivalente, unterschiedliche Wege der Aufgabenbewältigung möglich, und schließlich ist - insbesondere bei den in diesem Band fokussierten Teams - die Planung, Abstimmung, Steuerung und die notwendige Kommunikation zwischen den an der gemeinsamen Aufgabe Arbeitenden selbst ein zentraler Teil ihrer Arbeitstätigkeit (Interdependenz und Interaktion). Arbeitsgestaltung wird dabei immer mehr zu einem kontinuierlichen Teil der Arbeitsaufgabe selbst (u. a. Kötter und Volpert 1993).

Eine erste Konsequenz für die Arbeitsgestaltung aus dieser skizzierten Situation ist aus unserer Sicht: Je individualisierbarer die Arbeitsbedingungen und -situationen in solchen Projekt- oder Teamkontexten werden, d. h. je individueller die Bedingungen der Arbeit mit den Anforderungen, arbeitsbezogenen Belastungen und Ressourcen werden, umso weniger kann im Voraus hinreichend präzise vorgegeben werden, was in welcher Reihenfolge zu tun sein wird. Umso wichtiger wird es stattdessen, dass die Beschäftigten selbst in die Lage versetzt werden, betriebliche wie individuelle Bedürfnisse und Ansprüche an die Arbeitsgestaltung und Arbeitsorganisation zu reflektieren und zu formulieren. Dies gilt es auch für die Auslegung und Gestaltung des technischen Systems zu berücksichtigen, die hier nicht einschränkend sein sollte - die soziotechnische Design-Regel der ,minimal criticalspecification“ (vgl. Cherns 1987; Clegg 2000) beschreibt dieses Zielkriterium nach wie vor treffend.

Je weniger also einheitlich die Arbeitssysteme bzw. Arbeitsplätze gestaltbar sind, umso mehr müssen die Beschäftigten selbst - schon aus Selbstschutz, aber auch weil nur sie die genauen Bedingungen und Abläufe kennen und koordinieren müssen - über Grundkompetenzen der Arbeitsgestaltung („Gestaltungskompetenz“, vgl. Gerlmaier 2019a; Janneck und Hoppe 2018) und Kenntnisse über Regeneration und Erholung verfügen, um die evtl. gegebenen Handlungs- und Entscheidungsspielräume operativ zu nutzen oder bei Gestaltungsprozessen eine aktive Rolle einnehmen zu können.

Gleichzeitig sind solche Kompetenz-Voraussetzungen auch beim Management und den für die technische Infrastruktur Verantwortlichen $\mathrm{zu}$ fördern und $\mathrm{zu}$ entwickeln. Es bedarf deshalb insbesondere auch geeigneter Hilfsmittel, die z. B. Konstrukteure, 
IT- sowie Organisationsfachleute motivieren und befähigen, bereits bei ihren Automatisierungs- und Managementaktivitäten das Gestalten guter Arbeit zu berücksichtigen. Die betriebliche, die technische und die Arbeitsperspektive wird durch sie gleichermaßen in den Gestaltungsprozessen zu berücksichtigen sein (,organisationale Gestaltungskompetenz“, Gerlmaier 2019a, b; Gerlmaier und Latniak 2016). Arbeits- und Organisationsgestaltungsprozesse sind damit als interaktive Aushandlungen der daran Beteiligten zu begreifen.

Damit die Gestaltungskompetenzen auch praktisch wirksam werden können, ist vorausgesetzt, dass im Gestaltungsprozess diese Perspektiven angemessen eingebracht und auch berücksichtigt werden können: Der Gestaltungsprozess ist bei den angesprochenen Tätigkeiten kein einmaliger Vorgang mehr, der nach einem Durchgang unmittelbar zu einem abgeschlossenen Design oder einem fixen Endzustand führt. Gestaltungsprozesse müssen vielmehr einer Revision und Reflexion, d. h. einem verbesserungsorientierten Lernen der Nutzenden mit dem Arbeitssystem, gegenüber offen angelegt sein. Damit wäre der Anspruch an Gestaltungsprozesse, dass diese reflexiv, zyklisch und iterativ anzulegen und durchzuführen sind, um angemessenen Raum zu schaffen, aus den Erfahrungen der Beschäftigten bzw. der Ausführenden bei der Nutzung der Arbeitssysteme zu lernen. Dieses Prozessverständnis sehen wir als Weiterentwicklung der sog. ,Wendeltreppe der Arbeitsgestaltung', wie sie zunächst in den 1990er Jahren entwickelt wurde (u. a. Falck 1991; Kötter und Volpert 1993), welches allerdings die reflexiven Elemente noch stärker in den Fokus rückt (vgl. u. a. Lange und Longmuß 2015). Dies ist zudem eine Antwort auf die in vielen Bereichen zu beobachtende, geplante Unplanbarkeit', die sich als Folge zunehmender Markt- und Kundenöffnung in den Wertschöpfungs- und Dienstleistungsprozessen für die Ausführenden ergibt.

Dabei gilt weiterhin, dass das, was an Arbeit bewusst nach den arbeitswissenschaftlichen Humankriterien gestaltet werden kann, auch so zu gestalten ist - die Kenntnis und Berücksichtigung dieser Kriterien steht außer Frage. ${ }^{4}$ Ein , design by default ${ }^{*}$ gilt es zu vermeiden, das Arbeitsbedingungen quasi als Abfall- oder Nebenprodukt eines Prozessdesigns entstehen lässt und so häufig zu Zusatzaufwand bzw. weiteren Belastungen, dem Risiko ,interessierter Selbstgefährdung“ (Krause et al. 2015) oder mittelbar zu negativen Beanspruchungsfolgen beiträgt.

Hier gilt es zudem, weiterhin die unterschiedlichen Ebenen der Gestaltung zu unterscheiden (vgl. Luczak 1997), aber integriert zu betrachten und zu bearbeiten. Dabei verschieben sich die Gewichte der Ebenen bedingt durch die skizzierten Veränderungen:

\footnotetext{
${ }^{4}$ Beteiligung setzt operationalisierte und im Gestaltungsprozess genutzte arbeitswissenschaftliche Zielkriterien nicht außer Kraft, sondern ist der Modus in der betrieblichen oder überbetrieblichen Praxis, in dem sie ihre Wirksamkeit entfalten müssen. Gestaltungsprozesse ohne Zielkriterien sind blind; gleichzeitig bleiben diese Kriterien ohne geeignete Umsetzung durch die Handelnden in Gestaltungsprozessen leer.
} 
Neben 1) den individuellen, arbeitsplatzbezogenen Aspekten gilt es, 2) die Teamebene mit ihren Voraussetzungen zu berücksichtigen. Hier wäre - insbesondere für die Entwicklung von Ziel- und Bewertungskriterien - u. a. an Arbeiten aus den 1990er Jahren zur kollektiven Handlungsregulation (u. a. Weber 1997) anzuschließen, die beantworten können, wie vollständige Handlungen im Sinne der Handlungsregulationstheorie (vgl. Bergmann und Richter 1994) in Teamstrukturen gestaltet und möglich werden können.

Bedingt durch die Bedeutung der medienvermittelten Kommunikation kommen 3) die Teaminteraktion einerseits und andererseits die Einbindung der Teams in die Organisation und damit die Organisationsgestaltung als Kontext der Arbeitsgestaltung in den Blick. Die explizite Gestaltung der jeweiligen Schnittstellen im Team, zwischen unterschiedlichen Teams, sowie der Schnittstellen an den organisatorischen Grenzen ist ein Aspekt mit zunehmender praktischer Bedeutung für die Arbeitsgestaltung und insbesondere für die Belastungs- und Beanspruchungssituation, je mehr Personen an solchen ,Drehpunktpositionen“ (zwischen ,innen“ und ,außen' der jeweiligen Organisationseinheit) arbeiten, - wie z. B. mit externen Portalen oder IT-Systemen von Kunden oder Zulieferern.

Gestaltung muss in solchen medienvermittelten Arbeitszusammenhängen immer Arbeits- und Organisationsgestaltung sein und kann sich nicht allein auf die Gestaltung eines einzelnen Arbeitsplatzes oder eines einzelnen Prozessschrittes in einem Workflow beschränken (vgl. dazu u. a. Majchrzak 1997). Es gilt, konsequenter die Arbeit entlang des gesamten zusammenhängenden Arbeitsprozesses zu gestalten, denn Nutzende können in den Abläufen an unterschiedlichen Orten unterschiedlich davon betroffen sein, wenn z. B. am System bzw. am Ablauf von Prozessschritten (d. h. der Koordination der jeweiligen Aufgaben) etwas verändert wird.

Dies mag sich zunächst wie eine Binsenweisheit der Arbeitssystemgestaltung der 1970er Jahre anhören (vgl. u. a. Ulich 2011; zum soziotechnischen Systemansatz u. a. Bendel et al. 2020; Imanghaliyeva et al. 2020; Pasmore et al. 2018). Unter den skizzierten Bedingungen und technischen Möglichkeiten wird diese Perspektive aber immer wichtiger: Winby und Mohrman (2018) zeigten exemplarisch für Dialyseleistungen, dass eine zunehmende Anzahl von notwendigerweise Beteiligten zu berücksichtigen und in den Gestaltungsprozess einzubinden sind - sowohl um (wie im Beispiel) die Dienstleistungsqualität der Dialyse zu sichern, als auch um menschengerechte Arbeitsbedingungen zu schaffen. Wie dies praktisch jeweils realisiert werden kann, ist im konkreten Einzelfall zu klären.

Die dabei entstehenden Abhängigkeiten aus der Kooperation zwischen Mitarbeitenden als auch ,zwischen ihnen und den genutzten Ressourcen (Technik, Material, Information etc.)“ (Herrmann 2012, S. 19) führen dazu, dass die Arbeits- und Organisationsgestaltung sich verstärkt und bewusster als bisher mit der Interaktion und Kommunikation zwischen den Teammitgliedern und ihrer Umgebung befassen muss. Weiterführend ist es in diesem Zusammenhang, das betriebliche Informationssystem bzw. die genutzte technische Plattform als Infrastruktur zu begreifen (Pipek und Wulf 2009), die selbst wiederum in andere Systeme eingebettet ist (zur Verschachtelung der 
Systeme: vgl. Winter et al. 2014): Sie bietet den Nutzenden die Möglichkeit, neue Handlungsmöglichkeiten zu erschließen und ihre Kompetenzen weiter zu entwickeln (Herrmann 2012).

Damit kommt (4) ein „ecosystem“ (Pasmore et al. 2018), oder anders gesagt: die zusammenwirkenden ,Systeme von ineinander verschachtelten Arbeitssystemen" in den Fokus der Arbeits- und Organisationsgestaltung: „In short, work systems have become complex, technicologically enabled networked ecosystems that extend beyond an organization and its employees and are geographically dispersed." (Winby und Mohrman 2018, S. 3) Die Gestaltung der Arbeitsbedingungen wird bei Fortschreiten der technischen Vernetzung durch arbeitsplatz- und unternehmensexterne technische Systeme und Rahmensetzungen eingeschränkt bzw. zunehmend festgelegt: Je übergreifender und vernetzter die Arbeitsprozesse angelegt sind, desto häufiger werden solche Herausforderungen in den Arbeits- und Organisationsgestaltungsprozessen zu lösen sein.

Der Gestaltungsprozess ist letztlich so anzulegen, dass zentrale aufgabenbezogene Fragen möglichst frühzeitig im Kreis der an den Arbeitszusammenhängen Beteiligten geklärt und festgelegt werden können. Hierfür sind neue und effiziente Wege der Einbindung Betroffener und der Anlage von Gestaltungsprozessen zu erarbeiten, die diese Leerstelle der Gestaltung systematisch aufzufüllen im Stande ist.

\subsection{Beiträge und Beitragende zu diesem Sammelband}

Wir gehen mit der bisherigen Forschung zu Team- und Projektarbeit davon aus, dass diese kooperativen Arbeitsformen Potenziale bieten, um die oben skizzierten Herausforderungen einer als zunehmend komplex wahrgenommenen Arbeitswelt zu bewältigen - abhängig von der Aufgaben- oder Problemstellung und unter Einhaltung gewisser Anforderungen an die Arbeits- und Organisationsgestaltung. Mit den technologischen, organisatorischen und gesellschaftlichen Veränderungen ändern sich allerdings auch die Bedingungen, unter denen Projekt- und Teamarbeit heute stattfindet bzw. stattfinden kann.

Wenngleich die fortschreitende Digitalisierung, wie angedeutet, nicht der einzige Auslöser für die Veränderungen unserer Arbeitswelt ist, so waren es sicher gerade die damit verbundenen Entwicklungen, die das Bundesministerium für Bildung und Forschung (BMBF) veranlassten, den Förderschwerpunkt „Arbeit in der digitalisierten Welt“ zu initiieren. Mit dem Anspruch, bestehende Forschungslücken zu schließen sowie Gestaltungsspielräume in enger Zusammenarbeit von Wissenschaft und Wirtschaft zu finden und anwendungsnah zu nutzen, wurden insgesamt 29 Verbundprojekte mit Mitteln des Bundes und des europäischen Sozialfonds gefördert. Ziel war es, die ökonomischen und arbeitsbezogenen Potenziale der Digitalisierung für Unternehmen und Beschäftigte durch soziale Innovationen nutzbar zu machen. 
Zur Förderung von Vernetzung und Zusammenarbeit wurden vom koordinierenden Verbundprojekt ,TransWork' ${ }^{5}$ fünf Schwerpunktgruppen thematisch verwandter Projektverbünde gebildet. Dieser Sammelband enthält Beiträge aus der Schwerpunktgruppe „Projekt- und Teamarbeit in der digitalisierten Arbeitswelt“, in der sechs Verbundprojekte zusammengefasst waren, die sich mit Fragen der Organisation, Führung und Gestaltung von Arbeit, insbesondere kooperativer Arbeit beschäftigten. ${ }^{6}$ Die Koordination der Aktivitäten der Schwerpunktgruppe erfolgte durch das Institut für Arbeitswissenschaft der RWTH Aachen University ${ }^{7}$, das im Rahmen seines Teilvorhabens im TransWork-Projekt auch eigene Forschungsleistungen zum Themengebiet erbracht hat (FKZ: 02L15A162).

Aufgrund der anwendungsnahen Ausrichtung haben die durchgeführten Untersuchungen mehrheitlich Fall- und Feldstudiencharakter. An den Verbundprojekten waren Unternehmen unterschiedlicher Branchen und Größen beteiligt, wobei der Anteil kleiner und mittlerer Unternehmen in dieser Schwerpunktgruppe deutlich überwog $(66 \%)$. Unter den Betriebspartnern fanden sich u. a. mehrere Unternehmen der IT-Branche und der Metall- und Elektroindustrie, ein Hersteller von Maschinen und Anlagen, eine Unternehmensberatung sowie ein Schiffsbauunternehmen. ${ }^{8}$

In den Fallstudien wurden Beschäftigte aus unterschiedlichen Fachbereichen, Abteilungen und Hierarchieebenen befragt, es wurden Workshops und Gruppendiskussionen moderiert sowie Team- bzw. Projektsitzungen teilnehmend beobachtet. Ein Schwerpunkt lag dabei auf Projekten und Arbeitsaufgaben, die als komplex, interaktiv und wissensintensiv charakterisiert werden können und vorrangig kognitive, mentale und soziale Anforderungen an die Beteiligten stellen. Vor dem Hintergrund ihrer jeweiligen Disziplinen und der spezifischen Forschungsfragestellungen untersuchten die Forschergruppen die organisationalen, technologischen oder personalen Bedingungen unter

${ }^{5}$ Das Verbundprojekt „TransWork - Transformation der Arbeit durch Digitalisierung“ (FKZ: 02L15A160 ff.) wurde mit der wissenschaftlichen Begleitung des Förderschwerpunkts betraut. Übersichten über alle Schwerpunktgruppen und Projekte finden sich in übergreifenden Publikationen (siehe hierzu Bauer et al. 2019; Bauer et al. 2020). Die vier weiteren Schwerpunktgruppen, betrafen die Themenfelder 1) Assistenzsysteme und Kompetenzentwicklung, 2) Produktivitätsmanagement, 3) Arbeitsgestaltung im digitalen Veränderungsprozess sowie 4) Gestaltung vernetzt-flexibler Arbeit. Aus den Ergebnissen des Förderschwerpunkts werden weitere Publikationen im Springer-Verlag zu folgenden Themen veröffentlicht: Produktivitätsmanagement (s. Jeske und Lennings 2020), Gestaltung vernetzt flexibler Arbeit (s. Daum et al. 2020); Digitale Führung und Zusammenarbeit (s Antoni et al. 2021); Digitalisierung \& Pflege (s. Bleses et al. 2020; Kubek et al. 2020).

${ }^{6}$ Eine Auflistung der beteiligten Projekte findet sich oberhalb des Impressums zu diesem Band.

${ }^{7}$ Die Schwerpunktgruppe „Projekt- und Teamarbeit in der digitalisierten Arbeitswelt“ wurde von Prof. Dr. Susanne Mütze-Niewöhner, Markus Harlacher und bis Ende 2018 von Dr. Philipp M. Przybysz geleitet.

${ }^{8}$ Das TransWork-Teilvorhaben bildet hier aufgrund seiner Anlage als Begleitprojekt ohne Anwendungsunternehmen eine Ausnahme. 
denen Projekt- und Teamarbeit heute stattfindet, identifizierten Belastungsfaktoren, Anforderungen und Verbesserungspotenziale, entwickelten dabei auch Unterstützungsinstrumente und Lösungsansätze und leiteten schließlich auf der Grundlage ihrer Ergebnisse Empfehlungen für die Gestaltung menschengerechter und effektiver Arbeitsbedingungen bei Projekt- und Teamarbeit in digitalisierten Arbeitssystemen ab.

Die Beiträge, die im Folgenden kurz im Überblick dargestellt werden sollen, liefern in ihrer Gesamtheit Antworten auf folgende Fragen:

1. Welche Veränderungen der Arbeits- und Betriebsorganisation lassen sich beobachten und unter welchen Bedingungen findet intra- und interorganisationale Projekt- und Teamarbeit aktuell statt?

2. Wie wirken sich veränderte Technologien, Prozesse und Strukturen auf die Arbeitssituation der Beteiligten aus? Welche Herausforderungen gilt es zu bewältigen? Wo bestehen Gestaltungspielräume und -bedarfe?

3. Wie kann verteilte, virtuelle Zusammenarbeit so unterstützt werden, dass Prozessgewinne erzielt und -verluste vermieden werden können?

4. Welche Empfehlungen können für die Gestaltung, Organisation, Führung und Regulierung von Projekt- und Teamarbeit gegeben werden?

Der Sammelband startet mit einem Beitrag von Judith Neumer und Manuel Nicklich zu agilen Projektteams. Die Autor*innen thematisieren die „Fluidität“ und organisatorische Beschaffenheit von agilen Teams. Auf Grundlage ihrer Untersuchungen diskutieren sie wesentliche Gestaltungsaspekte, wie die Kundenintegration und die Einbettung in hierarchische Organisations- und Führungsstrukturen, und sie zeigen die daraus resultierenden Herausforderungen für die Selbstorganisation agiler Teams auf. Dabei wird u. a. deutlich, dass auch digitale Tools nur begrenzt in der Lage sind, räumliche Distanzen in solchen, verteilt arbeitenden Teams zu überbrücken.

Auch in der von Markus Harlacher, Verena Nitsch und Susanne Mütze-Niewöhner vorgestellten Studie zur Komplexität im Projektmanagement erweisen sich arbeitsorganisatorische Aspekte als zentrale Ansatzpunkte für die komplexitätsregulierende Arbeitsgestaltung. Zielgruppe der Untersuchung sind Projektmanager*innen, die in einer Onlinebefragung insbesondere Faktoren als besonders komplexitätstreibend benannten, die zu hohen psychischen Belastungen führen können. Dabei zeigten sich z. T. Unterschiede in Abhängigkeit des jeweils gewählten Managementansatzes (,,klassisch“, ,,agil“, „hybrid").

Erich Latniak und Jennifer Schäfer präsentieren Ergebnisse ihrer Analysen zur Belastungs- und Ressourcensituation operativer Führungskräfte virtueller Teams. Ihre Empfehlungen zielen darauf ab, Zeitdruck, Multitasking, Arbeitsunterbrechungen etc. zu reduzieren und die Nutzung persönlicher Ressourcen u. a. durch gezieltes Coaching zu stärken. Die Ergebnisse korrespondieren teilweise mit der Studie von Harlacher et al. in diesem Band und stützen insbesondere den in Abschn. 1.3.2 formulierten Bedarf, 
die Entwicklung von (Arbeits-)Gestaltungskompetenz in Unternehmen nachhaltig zu fördern.

Dem Belastungsfaktor „Zeitdruck“ widmen sich Ulrike Pietrzyk, Michael Gühne und Winfried Hacker in ihrem Beitrag. Sie stellen die Entwicklung eines Verfahrens zur Ermittlung nachhaltiger Zeitbedarfe für komplexe Wissens- und Innovationsarbeit vor, welche häufig in Team- und Projektzusammenhängen erbracht wird. Indem zukünftige Zeitbedarfe partizipativ und konsensual in Kleingruppen ermittelt werden, können typische Schwierigkeiten, wie Planungsfehlschlüsse und die individuelle Abhängigkeit der erfassten Zeitbedarfe, vermieden werden.

Valeria Bernardy, Rebecca Müller, Anna T. Röltgen und Conny H. Antoni richten ihren Fokus auf die Führung hybrider Formen virtueller Teams. Hierunter werden Teams verstanden, in denen nur ein Teil der Teammitglieder, z. B. aufgrund von Home-Office oder Dienstreisen, nicht vor Ort in der Betriebsstätte arbeiten. Aus den spezifischen Bedingungen resultieren neue Anforderungen an Führungskräfte und Teammitglieder, die es zu bewältigen gilt, um negative Auswirkungen auf die Effektivität und die Qualität der Teamarbeit zu vermeiden.

Anforderungen an Führungskräfte vollständig virtuell arbeitender Teams werden im Beitrag von Rebekka Mander, Frank Müller und Ulrike Hellert thematisiert. Infolge eingeschränkter Kommunikation und Wahrnehmung erhalten bei Führung auf Distanz vertrauensbildende Maßnahmen sowie die Gewährung von Zeit- und Handlungsspielräumen eine besondere Bedeutung. Die Autor*innen geben Einblicke in die Ergebnisse ihrer Erhebungen und leiten Empfehlungen für die Führungspraxis ab.

Digitalisierungsprojekte in der Produktentwicklung und -entstehung sind Gegenstand des Beitrags von Victoria Zorn, Julian Baschin, Nine Reining, David Inkermann, Thomas Vietor und Simone Kauffeld. Die Autor*innen plädieren für einen Gestaltungsansatz, der die Prozesse, die Methoden/Tools und die Kompetenzen der Beteiligten gleichermaßen in den Blick nimmt. Sie untermauern ihren Ansatz anhand von zwei Fallstudien im Maschinen- und Anlagenbau, die den Einsatz von Simulationssoftware in der länderübergreifenden Produktentwicklung und die Virtualisierung von Inbetriebnahmen zum Gegenstand hatten.

Abgerundet wird der Sammelband durch zwei Beiträge von Thomas Hardwig und Marliese Weißmann, die sich den Herausforderungen der Arbeitsgestaltung und -regulierung im Zusammenhang mit der Einführung und Nutzung von Kollaborationsplattformen widmen. Im Mittelpunkt des ersten Beitrags stehen die Erkenntnisse und Erfahrungen aus der Begleitung von drei Unternehmen bei der Suche nach dem digitalen Arbeitsplatz, d.h. der Einführung und Nutzung von geeigneten Kollaborationsplattformen. Die Autor*innen beschreiben anspruchsvolle Such-, Lern- und Entwicklungsprozesse der Unternehmen und empfehlen aufgrund dessen ein iteratives, agiles Vorgehen, um solche komplexen Anforderungen zu bewältigen. Im zweiten Beitrag identifizieren die Autor*innen die wesentlichen Charakteristika solcher Plattformen, diskutieren die Chancen und Risiken ihres Einsatzes und zeigen Gestaltungsspielräume 
auf, die von den betrieblichen Akteur*innen zur Aushandlung unternehmensspezifischer Lösungen genutzt werden können und sollten.

\section{Literatur}

Akin N, Rumpf J (2013) Führung virtueller Teams. Gruppendyn Organisationsberat 44:373-387. https://doi.org/10.1007/s11612-013-0228-9

Antoni CH (2000) Teamarbeit gestalten; Grundlagen, Analysen, Lösungen. Beltz, Weinheim

Antoni CH (2010) Interprofessionelle Teamarbeit im Gesundheitsbereich. Zeitschrift für Evidenz, Fortbildung und Qualität im Gesundheitswesen 104:18-24

Antoni CH (2016) Gruppenarbeit wirkungsvoll gestalten. In: Jöns I (Hrsg) Erfolgreiche Gruppenarbeit. Konzepte, Instrumente, Erfahrungen. Springer Gabler, Wiesbaden, S 13-24

Antoni CH (2017) Gruppen- und Teamarbeit. In: Spath D, Westkämper E, Bullinger H-J, Warnecke H-J (Hrsg) Neue Entwicklungen in der Unternehmensorganisation. Vieweg, Berlin, S $161-172$

Antoni $\mathrm{CH}$, Hellert U, Latniak E (in Vorbereitung) Digitale Führung und Zusammenarbeit. Springer Vieweg, Berlin

Baethge M, Oberbeck H (1986) Zukunft der Angestellten; Neue Technologien und berufliche Perspektiven in Büro und Verwaltung. Campus, Frankfurt a. M.

Bauer W, Stowasser S, Mütze-Niewöhner S, Zanker C, Brandl K-H (Hrsg) (2019) Arbeit in der digitalisierten Welt. Stand der Forschung und Anwendung im BMBF-Förderschwerpunkt. Frauenhofer IAO, Stuttgart

Bauer W, Mütze-Niewöhner S, Stowasser S, Zanker C, Müller N (in Vorbereitung) Arbeit in der digitalisierten Welt - Praxisbeispiele und Gestaltungslösungen aus dem BMBF-Förderschwerpunkt. Springer Vieweg, Berlin

Bendel A, Latniak E, Werner L (2020 i. E.) Bericht vom Workshop ,,agil - lean - soziotechnisch: Konzepte und Vorgehensweisen für Arbeits- und Organisationsgestaltung in Digitalisierungsprozessen“, BAuA, Dortmund, 01.10.2019. Z Arb Wiss 74:158-159. https://doi.org/10.1007/ s41449-020-00208-9

Berggren C (1991) Von Ford zu Volvo; Automobilherstellung in Schweden. Springer, Berlin

Bergmann B, Richter P (Hrsg) (1994) Die Handlungsregulationstheorie; Von der Praxis einer Theorie. Hogrefe, Göttingen

Bleses P, Busse B, Friemer A (2020) Digitalisierung der Arbeit in der Langzeitpflege als Veränderungsprojekt. Springer Vieweg, Berlin

BMAS, Bda, DGB, (2013) Gemeinsame Erklärung: Psychische Gesundheit in der Arbeitswelt. BMAS, Berlin

Boes A, Bultemeier A, Gül K, Kämpf T, Langes B, Lühr T, Marrs K, Ziegler A (2015) Zwischen Empowerment und digitalem Fließband: Das Unternehmen der Zukunft in der digitalen Gesellschaft. In: Boes A, Welpe I, Sattelberger T (Hrsg) Das demokratische Unternehmen. Neue Arbeits- und Führungskulturen im Zeitalter digitaler Wirtschaft. Haufe-Lexware GmbH \& Co. KG, Freiburg, S 57-76

Boos M, Hardwig T, Riethmüller M (2017) Führung und Zusammenarbeit in verteilten Teams. Hogrefe, Göttingen

Born A (2000) Gemeinsam oder einsam? Arbeitsorganisation im Dienstleistungssektor. Nordhause-Janz \& Pekruhl 2000:102-138

Braun T (2018) Kooperatives Verhalten in interorganisationalen Projekten; Eine konzeptionelle und empirische Weiterentwicklung des OCB-Ansatzes. Springer, Wiesbaden 
Braun T, Sydow J (2017) Projektmanagement und temporäres Organisieren. Kohlhammer, Stuttgart

Bredehöft F, Dettmers J, Hoppe A, Janneck M (2015) Individual work design as a job demand: the double-edged sword of autonomy. Psychol Everyday Act 8:13-26

Brödner P (1985) Fabrik 2000; Alternative Entwicklungspfade in die Zukunft der Fabrik. Nomos edition sigma, Berlin

Cherns A (1976) The principles of sociotechnical design. Hum Relat 29:783-792. https://doi. org/10.1177/001872677602900806

Cherns A (1987) Principles of sociotechnical design revisted. Hum Relat 40:153-161. https://doi. org/10.1177/001872678704000303

Clegg CW (2000) Sociotechnical principles for system design. Appl Ergon 31:463-477. https:// doi.org/10.1016/S0003-6870(00)00009-0

Cram WA, Newell S (2016) Mindful revolution or mindless trend? Examining agile development as a management fashion. Eur J Inform Syst 25:154-169. https://doi.org/10.1057/ejis.2015.13

Daum M, Wedel M, Zinke-Wehlwann C, Ulbrich H (Hrsg) (2020) Gestaltung vernetzt-flexibler Arbeit; Beiträge aus Theorie und Praxis für die digitale Arbeitswelt. Springer, Berlin

DIN EN ISO 10075 (2018) Ergonomische Grundlagen bezüglich psychischer Arbeitsbelastung. Beuth, Berlin

DIN EN ISO 6385 (2016) Grundsätze der Ergonomie für die Gestaltung von Arbeitssystemen. Beuth, Berlin

DIN EN ISO 9241-210 (2019) Prozess zur Gestaltung gebrauchstauglicher interaktiver Systeme. Beuth, Berlin

Eurofound (2015) Sechste Europäische Erhebung über die Arbeitsbedingungen 2015. Item „Eigenverantwortung des Teams“. https://www.eurofound.europa.eu/de/data/european-workingconditions-survey. Zugegriffen: 2. Juli 2020

Falck M (1991) Partizipative Systemgestaltung in Sozialen Organisationen. In: Brödner P, Simonis G, Paul H (Hrsg) Arbeitsgestaltung und partizipative Systementwicklung. VS Verlag, Wiesbaden, S 39-58

Gerlmaier A (2019a) Neue Gestaltungsoptionen oder Null-Puffer? In: Gerlmaier A, Latniak E (Hrsg) Handbuch psycho-soziale Gestaltung digitaler Produktionsarbeit. Gesundheitsressourcen stärken durch organisationale Gestaltungskompetenz. Springer Gabler, Wiesbaden, S 93-124

Gerlmaier A (2019b) Wer gestaltet die Arbeit im Zeitalter der Digitalisierung? In: Gerlmaier A, Latniak E (Hrsg) Handbuch psycho-soziale Gestaltung digitaler Produktionsarbeit. Gesundheitsressourcen stärken durch organisationale Gestaltungskompetenz. Springer Gabler, Wiesbaden, S $57-78$

Gerlmaier A, Latniak E (2007) Zwischen Innovation und täglichem Kleinkrieg; Arbeits- und Lernbedingungen bei Projektarbeit im IT-Bereich. In: Moldaschl M (Hrsg) Verwertung immaterieller Ressourcen. Nachhaltigkeit von Unternehmensführung und Arbeit III. Hampp, München, S 131-170

Gerlmaier A, Latniak E (Hrsg) (2011) Burnout in der IT-Branche; Ursachen und betriebliche Prävention. Asanger, Kröning

Gerlmaier A, Latniak E (2013) Psychische Belastungen in der IT-Projektarbeit - betriebliche Ansatzpunkte der Gestaltung und ihre Grenzen. In: Junghanns G, Morschhäuser M (Hrsg) Immer schneller, immer mehr. Psychische Belastung bei Wissens- und Dienstleistungsarbeit. Springer, Wiesbaden, S 165-193

Gerlmaier A, Latniak E (2016) Mehr Autonomie, mehr Resilienz oder mehr Gestaltungskompetenz? Neue Wege psycho-sozialer Arbeitsgestaltung im Industrie 4.0-Zeit- 
alter. Shortpaper bei der 14. Jahrestagung des Arbeitskreises Empirische Personal- und Organisationsforschung, 24./25. November 2016, Heinrich-Heine-Universität, Düsseldorf

Gerlmaier A, Latniak E (Hrsg) (2019) Handbuch psycho-soziale Gestaltung digitaler Produktionsarbeit. Springer Fachmedien Wiesbaden, Wiesbaden

Gesellschaft für Arbeitswissenschaft (GfA) (1999) Selbstverständnis der GfA e. V. GfA, Dortmund GPM (2015) Makroökonomische Vermessung der Projektwirtschaft in Deutschland. Deutsche Gesellschaft für Projektmanagement e. V., Nürnberg

Grote G (1997) Autonomie und Kontrolle; Zur Gestaltung automatisierter und risikoreicher Systeme. vdf Hochschulverl. an der ETH Zürich, Zürich

Hacker W (1994) Arbeitsanalyse zur prospektiven Gestaltung der Gruppenarbeit. In: Antoni CH (Hrsg) Gruppenarbeit in Unternehmen. Konzepte, Erfahrungen, Perspektiven. Beltz, Weinheim, S 49-80

Hacker W (1995) Arbeitstätigkeitsanalyse Analyse und Bewertung psychischer Arbeitsanforderungen. Asanger, Heidelberg

Hacker W (2009) Arbeitsgegenstand Mensch: Psychologie dialogisch-interaktiver Erwerbsarbeit; Ein Lehrbuch. Pabst, Lengerich

Hacker W (2017) Gesundheitsförderliche Arbeitsgestaltung in KMU. BePr. https://doi.org/10.3730 $7 / j .2365-7634.2017 .06 .07$

Hacker W (2018) Menschengerechtes Arbeiten in der digitalisierten Welt. Reihe Mensch-TechnikOrganisation. vdf-Hochschulverlag an der ETH Zürich, Zürich

Harmsen D-M, Weiß G, Georgieff P (1991) Automation im Geldverkehr; Wirtschaftliche und soziale Auswirkungen. VS Verlag, Wiesbaden

Heidling E (2018) Projektarbeit. In: Böhle F, Voß GG, Wachtler G (Hrsg) Handbuch Arbeitssoziologie. Band 2: Akteure und Institutionen. Springer, Wiesbaden, S 107-236

Heisig U, Littek W, Gondek H-D (1992) Arbeitsprozeß, Sozialbeziehung und Rationalisierung bei qualifizierten Dienstleistungstätigkeiten; Besonderheiten, Perspektiven, Themen. In: Littek W, Heisig U, Gondek H-D (Hrsg) Organisation von Dienstleistungsarbeit. Sozialbeziehungen und Rationalisierung im Angestelltenbereich. Nomos editionsigma, Berlin, S 9-32

Herrmann T (2012) Kreatives Prozessdesign; Konzepte und Methoden zur Integration von Prozessorganisation, Technik und Arbeitsgestaltung. Springer, Berlin

Hirsch-Kreinsen H (2015) Einleitung: Digitalisierung industrieller Arbeit. In: Hirsch-Kreinsen H, Ittermann P, Niehaus J (Hrsg) Digitalisierung industrieller Arbeit. Die Vision Industrie 4.0 und ihre sozialen Herausforderungen. Nomos editionsigma, Baden-Baden, S 9-30

Hirsch-Kreinsen H, Ramge U (1994) Qualifizierte Gruppenarbeit; Leistungspolitische Probleme und betriebliche Gestaltungsfelder. In: Moldaschl M, Schultz-Wild (Hrsg) Arbeitsorientierte Rationalisierung. Fertigungsinseln und Gruppenarbeit im Maschinenbau. Campus, Frankfurt a. M., S 33-49

Hodgson DE (2004) Project work: The legacy of bureaucratic control in the post-bureaucratic organization. Organization 11:81-100. https://doi.org/10.1177/1350508404039659

Hodgson DE, Briand L (2013) Controlling the uncontrollable: 'Agile' teams and illusions of autonomy in creative work. Work Employ Soc 27:308-325. https://doi. org/10.1177/0950017012460315

Höge T (2019) Workplace flexibility and employee well-being; proposing a life-conduct perspective on subjectified work. Psychol Everyday Act 12:9-19

Imai M (1993) Kaizen; Der Schlüssel zum Erfolg der Japaner im Wettbewerb. Ullstein, Berlin

Imanghaliyeva AA, Thompson P, Salmon P, \& Stanton NA (2020) A synthesis of sociotechnical principles for system design. In: Rebelo F, Soares MM (Hrsg) Advances in ergonomics in design. Proceedings of the AHFE 2019 International Conference on Ergonomics in Design, July 24-28, 2019, Washington D.C., USA, S 665-676 
Institut DGB-Index Gute Arbeit (2016) Wie die Beschäftigten die Arbeitsbedingungen in Deutschland beurteilen: Mit dem Themenschwerpunkt: Die Digitalisierung der Arbeitswelt - Eine Zwischenbilanz aus der Sicht der Beschäftigten. DGB-Index Gute Arbeit - Der Report 2016, Berlin

Jeske T, Lennings F (Hrsg) (2020) Produktivitätsmanagement 4.0; Praxiserprobte Vorgehensweisen zur Nutzung der Digitalisierung in der Industrie. Springer Vieweg, Berlin

Janneck M, Hoppe A (Hrsg) (2018) Gestaltungskompetenzen für gesundes Arbeiten; Arbeitsgestaltung im Zeitalter der Digitalisierung. Springer, Berlin

Joshi A, Roh H (2009) The role of context in work team diversity research; a meta-analytic review. Acad Manag J 52:599-627

Jöns I (Hrsg) (2008) Erfolgreiche Gruppenarbeit. Gabler GWV-Fachverlage, Wiesbaden

Kalkowski P (2013) Projekte (Projektarbeit, Projektmanagement). In: Hirsch-Kreinsen H, Minssen H (Hrsg) Lexikon der Arbeits- und Industriesoziologie. Nomos, Baden-Baden, S 400-404

Kalkowski P, Mickler O (2009) Antinomien des Projektmanagements; Eine Arbeitsform zwischen Direktive und Freiraum. Nomos, Baden-Baden

Kalkowski P, Mickler O (2015) Kooperative Produktentwicklung; Fallstudien aus der Automobilindustrie, dem Maschinenbau und der IT-Industrie. Nomos, Baden-Baden

Kauffeld S (2001) Teamdiagnose. Hogrefe, Göttingen

Kauffeld S, Schulte EM (2019) Teams und ihre Entwicklung. In: Kauffeld S (Hrsg) Arbeits-, Organisations- und Personalpsychologie für Bachelor (3. überarbeitete Aufl.). Springer, Berlin, S 211-236

Kauffeld S, Handke L, Straube J (2016) Verteilt und doch verbunden: Virtuelle Teamarbeit. Gruppe. Interaktion. Organisation. Z Angew Organ Psychol 47(1):43-51

Kern H, Schumann M (1986) Das Ende der Arbeitsteilung? Rationalisierung in der industriellen Produktion; Bestandsaufnahme, Trendbestimmung. Beck, München

Kirchner S, Oppen M (2007) Das Ende der Reorganisationsdynamik? High Performance Work Practices als Muster der Reorganisation in Deutschland. Discussion Paper SP III 2007-103, Wissenschaftszentrum Berlin

Kötter W (2002) Projektarbeit - (k)ein Thema für die Arbeitspsychologie? In: Moldaschl M (Hrsg) Neue Arbeit - neue Wissenschaft der Arbeit? Festschrift zum 60. Geburtstag von Walter Volpert. Asanger, Heidelberg, S 399-416

Kötter W, Volpert W (1993) Arbeitsgestaltung als Arbeitsaufgabe - ein arbeitspsychologischer Beitrag zu einer Theorie der Gestaltung von Arbeit und Technik. Z Arb Wiss 47:129-140

Krause A, Baeriswyl S, Berset M, Deci N, Dettmers J, Dorsemagen C, Meier W, Schraner S, Stetter B, Straub L (2015) Selbstgefährdung als Indikator für Mängel bei der Gestaltung mobilflexibler Arbeit. Wirtschaftspsychologie 4-2014(1-2015):49-59

Kubek V, Velten S, Eierdanz F, Blaudszun-Lahm A (2020) Digitalisierung in der Pflege; Zur Unterstützung einer besseren Arbeitsorganisation. Springer Vieweg, Berlin

Kuhlmann M, Schumann M (2015) Digitalisierung fordert Demokratisierung der Arbeitswelt heraus. In: Hoffmann R, Bogedan C (Hrsg) Arbeit der Zukunft. Möglichkeiten nutzen Grenzen setzen. Campus, Frankfurt a. M., S 122-141

Lange K, Longmuß J (2015) 6.3 Das PaGIMO-Veränderungsmodell. In: Zink KJ, Kötter W, Longmuß J \& Thul M (Hrsg) Veränderungsprozesse erfolgreich gestalten. Springer Vieweg, Berlin, S 169-173

Latniak E (2013) Leitideen der Rationalisierung und der demografische Wandel; Konzepte und Herausforderungen. In: Hentrich J, Latniak E (Hrsg) Rationalisierungsstrategien im demografischen Wandel. Handlungsfelder, Leitbilder und Lernprozesse. Springer Gabler, Wiesbaden, S 27-57 
Leimeister JM, Shkodran Z, Durward D, Blohm I (2016) Systematisierung und Analyse von Crowd-Sourcing-Anbietern und Crowd-Work-Projekten. Hans Böckler Stiftung, Düsseldorf

Lipnack J, Stamps J (1998) Virtuelle Teams; Projekte ohne Grenzen. Teambildung, virtuelle Orte, intelligentes Arbeiten, Vertrauen in Teams. Ueberreuter, Wien

Luczak H (Hrsg) (1997) Handbuch Arbeitswissenschaft. Schäffer-Poeschel, Stuttgart

Lutz B (1987) Das Ende des Technikdeterminismus und die Folgen: soziologische Technikforschung vor neuen Aufgaben und neuen Problemen. In: Lutz B (Hrsg) Technik und sozialer Wandel. Verhandlungen d. 23. Dt. Soziologentages in Hamburg 1986. Campus, Frankfurt, S 34-52

Majchrzak A (1997) What to do when you can't have it all; toward a theory of sociotechnical dependencies. Hum Relat 50:535-566. https://doi.org/10.1023/A:1016939819110

Malorny C (2014) Total Quality Management als Grundlage für die Entwicklung der Unternehmenskultur. In: Pfeifer T, Schmitt R (Hrsg) Masing Handbuch Qualitätsmanagement. Hanser, München, S 1042-1055

Manager Monitor (2017) Umfragen und Stimmungsbilder zu aktuellen Themen aus Steuerpolitik, Sozialpolitik, Arbeitspolitik, Europapolitik und Management. Ausgabe 01/2017 vom 07. Februar 2017. https://www.ula.de/wp-content/uploads/2017/02/20170208-manager-monitor. pdf. Zugegriffen: 4. Dez. 2019

Minssen H (2019) Dienstleistungsarbeit. In: Minssen H (Hrsg) Arbeit in der modernen Gesellschaft. Eine Einführung. Springer, Wiesbaden, S 143-158

Moore PV (2018) Tracking affective labour for agility in the quantified workplace. Body \& Society 24:39-67. https://doi.org/10.1177/1357034X18775203

Mütze-Niewöhner S, Schlick CM, Luczak H (2018) Gruppen- und Teamarbeit. In: Schlick CM, Bruder R, Luczak H (Hrsg) Arbeitswissenschaft. Springer, Berlin, S 681-728

Mütze-Niewöhner S, Nitsch V (2020) Arbeitswelt 4.0. In: Frenz W (Hrsg) Handbuch Industrie 4.0: Recht, Technik, Gesellschaft. Springer, Berlin, S 1187-1217

Muzio D, Hodgson D, Faulconbridge J, Beaverstock J, Hall S (2011) Towards corporate professionalization: the case of project management, management consultancy and executive search. Curr Sociol 59:443-464. https://doi.org/10.1177/0011392111402587

Nandram S, Koster N (2014) Organizational innovation and integrated care: lessons from Buurtzorg. J Integr Care 22:174-184. https://doi.org/10.1108/JICA-06-2014-0024

Nordhause-Janz J, Pekruhl U (Hrsg) (2000) Arbeiten in neuen Strukturen? Partizipation, Kooperation, Autonomie und Gruppenarbeit in Deutschland. Hampp, München

Oberbeck H (2013) Dienstleistungsarbeit. In: Hirsch-Kreinsen H, Minssen H (Hrsg) Lexikon der Arbeits- und Industriesoziologie. Nomos, Baden-Baden, S 103-106

Pasmore W, Winby S, Mohrman SA, Vanasse R (2018) Reflections: sociotechnical systems design and organization change. J Change Manag 19:67-85. https://doi.org/10.1080/14697017.2018.1 553761

Pfeiffer S, Sauer S, Ritter T (2014) Agile Methoden als Werkzeug des Belastungsmanagements? Eine arbeitsvermögensbasierte Perspektive. Arbeit 23. https://doi.org/10.1515/arbeit-2014-0206

Pipek V, Wulf V (2009) Infrastructuring: toward an integrated perspective on the design and use of information technology. JAIS 10:447-473. https://doi.org/10.17705/1jais.00195

Pruijt H (2003) Teams between neo-taylorism and anti-taylorism. Econ Ind Democracy 24:77-101. https://doi.org/10.1177/0143831X03241004

Raehlmann I (2017) Voraussetzungen der Entwicklung und Anwendung von Technik im Arbeitsprozess; Theoretische Ansätze und praktische Umsetzung. Z Arb Wiss 71:120-127. https://doi. org/10.1007/s41449-017-0053-z 
Ries BC, Diestel S, Shemla M, Liebermann SC, Jungmann F, Wegge J, Schmidt K-H (2016) age diversityand team effectiveness. In: Schlick CM, Frieling E, Wegge J (Hrsg) Age-differentiated work systems. Springer, Berlin, S 89-118

Rothe I, Wischniewski S, Tegtmeier P, Tisch A (2019) Arbeiten in der digitalen Transformation - Chancen und Risiken für die menschengerechte Arbeitsgestaltung. Z Arb Wiss 73:246-251. https://doi.org/10.1007/s41449-019-00162-1

Rump J, Schabel F, Alich D, Groh S (2010) Betriebliche Projektwirtschaft; Eine Vermessung. Eine empirische Studie des Instituts für Beschäftigung und Employability (IBE) im Auftrag von HAYS. HAYS, Mannheim

Schattenhofer K (2006) Teamarbeit jenseits der Idealisierung - eine Untersuchung. In: Edding C, Kraus W (Hrsg) Ist der Gruppe noch zu helfen? Gruppendynamik und Individualisierung. Budrich, Opladen, S 77-93

Seymour T, Hussein S (2014) The history of project management. IJMIS 18:233. https://doi. org/10.19030/ijmis.v18i4.8820

Snyder JR (1987) Modern project management: how did we get here-where do we go? Proj Manag J 18:28-29

Som O, Jäger A (2012) Qualität auf dem Vormarsch; Aktuelle Trends im Einsatz und in der Nutzung innovativer Organisationskonzepte. Mitteilungen aus der PI-Erhebung, Nr. 62, FhGISI, Karlsruhe

Staab P, Prediger LJ (2019) Digitalisierung und Polarisierung; Eine Literaturstudie zu den Auswirkungen des digitalen Wandels auf Sozialstruktur und Betriebe. FGW, Düsseldorf

Stahl GK, Maznevski ML, Voigt A, Jonsen K (2010) Unraveling the effects of cultural diversity in teams: a meta-analysis of research on multicultural work groups. J Int Bus Stud 41:690-709. https://doi.org/10.1057/jibs.2009.85

Stewart GL (2006) A meta-analytic review of relationships between team design features and team performance. J Manag 32:29-55. https://doi.org/10.1177/0149206305277792

Sträter O (2019) Wandel der Arbeitsgestaltung durch Digitalisierung. Z Arb Wiss 73:253-260

Sydow J (1985) Der soziotechnische Ansatz der Arbeits- und Organisationsgestaltung; Darstellung, Kritik, Weiterentwicklung. Campus, Frankfurt

Trist EL, Bamforth KW (1951) Some social and psychological consequences of the longwall method of coal-getting. Hum Relat 4:3-38. https://doi.org/10.1177/001872675100400101

Ulich E (2011) Arbeitspsychologie. vdf Hochschulverl. an der ETH Zürich. Schäffer-Poeschel, Zürich

Wageman R, Gardner H, Mortensen M (2012) The changing ecology of teams; New directions for teams research. J Organiz Behav 33:301-315. https://doi.org/10.1002/job.1775

Warner M, Witzel M (2004) Managing in virtual organizations. Thomson Learning, London

Weber WG (1997) Analyse von Gruppenarbeit; Kollektive Handlungsregulation in soziotechnischen Systemen. Teilw. zugl.: Zürich, Eidgenössische Techn. Hochsch., Habil.-Schr., 1996. Huber, Bern

Weber WG (1999) Gruppenarbeit in der Produktion. In: Zölch M, Weber WG, Leder L (Hrsg) Praxis und Gestaltung kooperativer Arbeit. vdf Hochschulverl. an der ETH Zürich, Zürich, S $13-69$

Wegge J (2004) Führung von Arbeitsgruppen. Hogrefe, Göttingen

Winby S, Mohrman SA (2018) Digital sociotechnical system design. J Appl Behav Sci 54:399423. https://doi.org/10.1177/0021886318781581

Windelband L (2014) Zukunft der Facharbeit im Zeitalter „Industrie 4.0“. JOTED 2:138-160

Winter S, Berente N, Howison J, Butler B (2014) Beyond the organizational 'container': Conceptualizing 21st century sociotechnical work. Inform Organ 24:250-269. https://doi. org/10.1016/j.infoandorg.2014.10.003 
Womack JP, Jones DT, Roos D (1992) Die zweite Revolution in der Autoindustrie; Konsequenzen aus der weltweiten Studie aus dem Massachusetts Institute of Technology. Campus, Frankfurt a. M.

Open Access Dieses Kapitel wird unter der Creative Commons Namensnennung 4.0 International Lizenz (http://creativecommons.org/licenses/by/4.0/deed.de) veröffentlicht, welche die Nutzung, Vervielfältigung, Bearbeitung, Verbreitung und Wiedergabe in jeglichem Medium und Format erlaubt, sofern Sie den/die ursprünglichen Autor(en) und die Quelle ordnungsgemäß nennen, einen Link zur Creative Commons Lizenz beifügen und angeben, ob Änderungen vorgenommen wurden.

Die in diesem Kapitel enthaltenen Bilder und sonstiges Drittmaterial unterliegen ebenfalls der genannten Creative Commons Lizenz, sofern sich aus der Abbildungslegende nichts anderes ergibt. Sofern das betreffende Material nicht unter der genannten Creative Commons Lizenz steht und die betreffende Handlung nicht nach gesetzlichen Vorschriften erlaubt ist, ist für die oben aufgeführten Weiterverwendungen des Materials die Einwilligung des jeweiligen Rechteinhabers einzuholen.

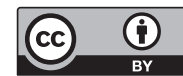

